\title{
Universal formulation of central-moments-based lattice Boltzmann method with external forcing for the simulation of multiphysics phenomena
}

DOI:

$10.1063 / 1.5124719$

\section{Document Version}

Accepted author manuscript

Link to publication record in Manchester Research Explorer

Citation for published version (APA):

De Rosis, A., Huang, R., \& Coreixas, C. (2019). Universal formulation of central-moments-based lattice Boltzmann method with external forcing for the simulation of multiphysics phenomena. Physics of Fluids (1994-present), 31, 1. [117102]. https://doi.org/10.1063/1.5124719

\section{Published in:}

Physics of Fluids (1994-present)

\section{Citing this paper}

Please note that where the full-text provided on Manchester Research Explorer is the Author Accepted Manuscript or Proof version this may differ from the final Published version. If citing, it is advised that you check and use the publisher's definitive version.

\section{General rights}

Copyright and moral rights for the publications made accessible in the Research Explorer are retained by the authors and/or other copyright owners and it is a condition of accessing publications that users recognise and abide by the legal requirements associated with these rights.

\section{Takedown policy}

If you believe that this document breaches copyright please refer to the University of Manchester's Takedown Procedures [http://man.ac.uk/04Y6Bo] or contact uml.scholarlycommunications@manchester.ac.uk providing relevant details, so we can investigate your claim.

\section{OPEN ACCESS}




\title{
Universal formulation of central-moments-based lattice Boltzmann method with external forcing for the simulation of multiphysics phenomena
}

\author{
Alessandro De Rosis, ${ }^{1,}$ a) Rongzong Huang, ${ }^{2,3, b)}$ and Christophe Coreixas ${ }^{4, c)}$ \\ 1) Department of Mechanical, Aerospace and Civil Engineering, The University of Manchester, Manchester M13 9PL, \\ $U K$ \\ ${ }^{2)}$ School of Mechanical Engineering, Shanghai Jiao Tong University, 200240 Shanghai, \\ China \\ ${ }^{3)}$ Institute of Aerodynamics and Fluid Mechanics, Technical University of Munich, 85748 Garching, \\ Germany \\ 4) Department of Computer Science, University of Geneva, 1204 Geneva, Switzerland
}

(Dated: 29 October 2019)

The cascaded or central-moments-based lattice Boltzmann method (CM-LBM) is a robust alternative to the more conventional Bhatnagar-Gross-Krook (BGK)-LBM for the simulation of high-Reynolds number flows. Unfortunately, its original formulation makes its extension to a broader range of physics quite difficult. In addition, it relies on CMs that are derived in an adhoc manner, i.e., by mimicking those of the Maxwell-Boltzmann distribution to ensure their Galilean invariance a posteriori. The present work aims at tackling both issues by deriving Galilean invariant CMs in a systematic and a priori manner thanks to the Hermite polynomial expansion framework. More specifically, the proposed formalism fully takes advantage of the D3Q27 discretization by relying on the corresponding set of 27 Hermite polynomials (up to the sixth-order) for the derivation of both the discrete equilibrium state and the forcing term in an a priori manner. Furthermore, while keeping the numerical properties of the original CM-LBM, the present work leads to a compact and simple algorithm, representing a universal methodology based on CMs and external forcing within the lattice Boltzmann framework. To support these statements, mathematical derivations and a comparative study with four other forcing schemes are provided. The universal nature of the proposed methodology is eventually proved through the simulation of single phase, multiphase (using both pseudo-potential and color-gradient formulations), as well as, magnetohydrodynamic flows.

\section{INTRODUCTION}

Nowadays, the lattice Boltzmann method (LBM) is a consolidated approach to simulate fluid flows ${ }^{1-10}$. The basic idea is to model the fluid through populations (or distributions) of fictitious particles moving along the links of a fixed Cartesian lattice. The space and time evolution of the distributions is predicted by solving the lattice Boltzmann equation that involves a collide-and-stream process, where the collision stage retains the flow physics. Then, a proper treatment of the collision process is instrumental to obtain accurate predictions of the fluid dynamics.

The impressive popularity of the LBM mainly stems from the intrinsic simplicity of the Bhatnagar-Gross-Krook (BGK) collision operator ${ }^{11}$. In short, all the populations are forced to relax (with a common unique rate) towards a discrete equilibrium state derived by applying a Gauss-Hermite quadrature to the continuous Maxwellian distribution ${ }^{12}$. Unfortunately, it is well known to be prone to numerical instability in the low-viscosity regime, thus becoming unsuitable for the prediction of turbulent flows.

About twenty years ago, entropic LBMs were introduced as a way to tackle this stability issue ${ }^{13-15}$. The derivation of such LBMs is based on H-theorem ${ }^{16}$, and it mainly consists in solving a minimization problem, at each grid node and for each time step, in order to ensure the growth over time of a predefined discrete entropy function. This methodology has led to great improvements in terms of numerical stability, and especially in under-resolved conditions, which was latter

\footnotetext{
a)Electronic mail: alessandro.derosis@manchester.ac.uk

b) Electronic mail: rongzong.huang@tum.de

c) Electronic mail: christophe.coreixas@unige.ch
}

explained by its subgrid scale properties ${ }^{17}$. To find a better trade-off between stability and CPU time, analytical approximations of the minimization problem was recently proposed $^{18,19}$. In the end, both methodologies were shown to be successful at simulating a large panel of very different phenomena $^{20-24}$.

Another popular alternative is represented by the multiple-relaxation-time (MRT) LBM, that suggests to perform the collision in a space of raw moments $(\mathrm{RMs})^{25-28}$. While second-order ones are related to the flow physics and relax with a frequency directly linked to the fluid kinematic viscosity, higher-order moments are related to non-hydrodynamic modes ${ }^{29,30}$, and their relaxation rates can be considered as free parameters. The MRT formulation allows to directly tune the relaxation frequencies related to these high-order contributions, eventually leading to an improved stability of the resulting LBM. In addition, the original MRT-LBM further increases the dissipation of acoustic waves, hence reducing their impact on the surrounding fluid dynamics ${ }^{31,32}$. Usually, both BGK and MRT LBMs relax to equilibrium states derived through a second-order truncated Taylor expansion in the local Mach number of the above-mentioned continuous Maxwellian distribution. As a consequence, these are Galilean invariant only up to the second-order meaning that velocity-dependent error of at least third-order are present in the macroscopic equations derived from the Chapman-Enskog expansion ${ }^{33}$. Unfortunately, the lack of Galilean invariance at higher-orders leads to numerical instability in the low viscous regime ${ }^{34,35}$.

To cope with this problem, Geier et al. ${ }^{36}$ proposed LBMs based on the concept of central moments (CMs), the latter being obtained by shifting the lattice directions by the local fluid velocity. In the actual practice, it is more convenient to operate in terms of RMs, thus requiring a 
transformation between the two types of moments. This is achieved by using binomial formulas, that generate central moments of a certain order as a function of lower-order RMs only $^{37}$. Consequently, the collision in the CM space shows a pyramidal hierarchical topology, where the post-collision state of $\mathrm{CMs}$ is constructed starting from the lowest order, and then proceeding in ascending sequence, hence the name "cascaded" lattice Boltzmann method (CLBM). Undoubtedly, the CLBM drastically outperforms both BGK and MRT in terms of stability ${ }^{36,38-58}$. The latter point can partially be explained by the positive hyperviscosity naturally introduced by CM-LBMs, and that can further be adjusted, through fine tuning of relaxation times related to high-order $\mathrm{CMs}$, in order to find the best compromise between stability and accuracy ${ }^{59}$.

An important step in the design of the CLBM is the match between continuous and discrete central moments, that enforces the Galilean invariance at all orders accordingly to the considered velocity discretization. In other words, CMs are forced to relax to the equilibrium state of the continuous Maxwellian distribution, rather than to its discrete counterpart. Consequently, this methodology assumes that equilibrium CMs are unchanged by the velocity discretization of the Boltzmann equation, which might not be true depending on both equilibrium CMs of interest and on the considered lattice of discrete velocities. In addition, the choice of the set of moments towards which equilibrium CMs are assumed to relax is not known in an a priori manner in the above methodology. This prevents any systematic derivation of CM-LBMs, especially, for high-order lattices whose discrete velocities propagate toward to the second layer of neighboring nodes (or even farther).

More recently, we approached central moments from a different viewpoint ${ }^{60-63}$. Given a certain lattice, our methodology consists of building a transformation matrix allowing us to move from the space of populations to the one of central moments and vice versa. The resultant algorithm loses the above-mentioned pyramidal cascaded structure and, as a consequence, it can be interpreted as a "non-cascaded" way to apply the collision step in CM space $^{64}$. In addition, this methodology is based on CMs computed from the polynomial expansion of populations instead of those derived from the continuous ones. Thus, one does not need anymore to choose which continuous CMs should be kept for the collision step. Consequently, the proposed approach allows the derivation of CM-LBMs for any lattice of discrete velocities in a straightforward manner. This was thoroughly demonstrated by successfully recovering different sets of governing equations with this approach, hence allowing the simulation of a rich variety of physics problems such as shallow waters ${ }^{65}$, magnetohydrodynamic ${ }^{66}$, and multicomponent flows ${ }^{67}$ among others.

Nonetheless, it is important to understand that the above attempt to derive a CMs-based scheme in the D3Q27 space was relying on the classical second-order truncated equilibrium state ${ }^{61}$. Even if the stability of the algorithm was greatly improved, it involved a huge computational $\operatorname{cost}^{48}$. This is mainly due to the use of an improper (or incomplete) equilibrium state, which eventually leads to a non-negligible number of non-zero velocity-dependent equilibrium CMs. Indeed, as pointed out by Malaspinas ${ }^{68}$ and Coreixas et al. ${ }^{35,69}$, the full potential of the D3Q27 velocity space can only be achieved by employing the correct (or complete) equilibrium populations accounting for the whole set of 27 Hermite polynomials. By including these high-order polynomials, the methodology outlined in ${ }^{61}$ was proven to lead to Galilean invariant CMs that were used for the derivation of a force-free D3Q27-CM-LBM ${ }^{70}$. This formulation was further shown to recover the behavior of the original cascaded LBM by only relying on the correct set of Hermite polynomials. Since this family of polynomials is tightly linked to the velocity discretization of the Boltzmann equation $^{12,71,72}$, the correct set of Hermite polynomials is known in an a priori way, hence ensuring its consistency for any kind of lattices.

When it comes to external or internal forces, it is well known that they are ubiquitous in many fluid systems. Indeed, gravity, Coriolis or Lorentz forces are just few examples of the fields that a fluid might undergo. It is then of paramount importance to properly take into account these forces in the lattice Boltzmann framework. To meet this need, a seminal contribution has been proposed by Guo et $a l .{ }^{73}$ in 2002 , where the authors analyzed the discrete lattice effects on the forcing term in the BGK-LBM. Later, Guo \& Zheng ${ }^{74}$ extended the approach to the MRT-LBM. Concerning the adoption of CMs, Premnath \& Banerjee derived forcing schemes based on the cascaded approach for two-and three-dimensional simulations ${ }^{55,56}$. In particular, they approximated the forcing term according to the formula in the pioneering work by He et al. ${ }^{75}$. In 2017, this forcing term has been adopted by Fei \& Luo ${ }^{47}$ within a framework where equilibrium CMs were computed from the continuous Maxwellian distributions. The same force treatment has been adopted in De Rosis ${ }^{62}$, where equilibrium CMs were derived from the classical second-order-truncated expansion. More recently, it has been demonstrated that the forcing term can be written as a Hermite polynomial expansion up to the fourth-order in the D2Q9 space $^{76}$, where the well-known formula by Guo et al. ${ }^{73}$ is recovered if Hermite polynomials of order higher than two are neglected.

In this paper, we derive a three dimensional CMs-based model with external forcing in an a priori manner for the simulation of multiphysics flows. Following the idea originally proposed by Shan et $a l^{12}$, and further adopted for the D2Q9 space by Huang et al. ${ }^{76}$, the forcing term is written as an expansion of Hermite polynomials. By adopting the very same set of Hermite polynomials for both the equilibrium state and the forcing term, it is thoroughly shown that corresponding CMs become Galilean invariant, i.e., all remaining error terms depending on the mean flow vanish. As a consequence, the number of non-zero CMs of the forcing term drops to nine, which drastically simplify its implementation.

The rest of the paper is organized as follows. The adopted methodology is first devised in Sec. II, and the impact of high-order Hermite polynomials on CMs of the forcing term is thoroughly quantified. In Sec. III, several numerical experiments of increasing complexity highlight the consistency, accuracy and universality of the proposed model. A comparative study with four other forcing schemes is also provided to further evaluate the numerical properties of the present approach, as well as, the impact of the truncation order of the Hermite polynomial expansion for both the equilibrium and the forcing term. Some conclusions 
are finally drawn in Section IV. For the sake of completeness, details regarding Hermite polynomials, the D2Q9-CM-LBM with external forcing, the lattice Boltzmann method for the magnetic field, and the CMs-based color-gradient method are reported in Apps. A, B, C, and D, respectively. Moreover, it is recalled in App. E that the CMs-based LBM can be interpreted as a general MRT LBM, where RMs are obtained as a particular case of the approach outlined in the next section.

\section{LATTICE BOLTZMANN SCHEMES WITH HIGHER-ORDER HERMITE POLYNOMIALS}

Let us consider an Eulerian basis $\boldsymbol{x}=[x, y, z]$ and the D3Q27 space $^{1}$. The lattice Boltzmann equation predicts the space and time evolution of the particle distribution functions $\left|f_{i}\right\rangle=\left[f_{0}, \ldots, f_{i}, \ldots, f_{26}\right]^{\top}$ that collide and stream along the generic link $i=0 \ldots 26$ with the discrete velocities $\boldsymbol{c}_{i}=\left[\left|c_{i x}\right\rangle,\left|c_{i y}\right\rangle,\left|c_{i z}\right\rangle\right]$ defined as

$$
\begin{aligned}
& \left|c_{i x}\right\rangle=[0, \quad 1,-1, \quad 0, \quad 0, \quad 0, \quad 0, \quad 1,-1, \quad 1,-1, \quad 1,-1, \quad 1,-1, \quad 0, \quad 0, \quad 0, \quad 0, \quad 1,-1, \quad 1,-1, \quad 1,-1, \quad 1,-1]^{\top},
\end{aligned}
$$

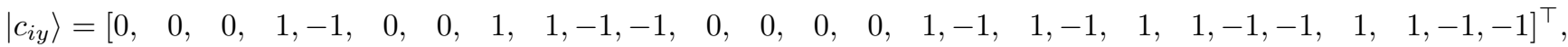

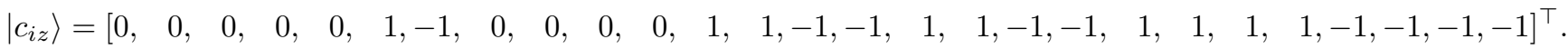

Let us employ the symbols $|\bullet\rangle$ and $T$ to denote a column vector and the transpose operator, respectively. Within the BGK approximation ${ }^{11}$, the numerical discretization of the lattice Boltzmann equation with external forcing reads as follows:

$$
\begin{aligned}
f_{i}\left(\boldsymbol{x}+\boldsymbol{c}_{i}, t+1\right)=f_{i}(\boldsymbol{x}, t) & +\omega\left[f_{i}^{e q}(\boldsymbol{x}, t)-f_{i}(\boldsymbol{x}, t)\right] \\
& +\left(1-\frac{\omega}{2}\right) \mathcal{F}_{i}(\boldsymbol{x}, t),
\end{aligned}
$$

where the lattice unit system was assumed. As usual, this numerical scheme can be divided into two parts, i.e., collision $^{73}$ :

$$
\begin{aligned}
f_{i}^{\star}(\boldsymbol{x}, t)=f_{i}(\boldsymbol{x}, t) & +\omega\left[f_{i}^{e q}(\boldsymbol{x}, t)-f_{i}(\boldsymbol{x}, t)\right] \\
& +\left(1-\frac{\omega}{2}\right) \mathcal{F}_{i}(\boldsymbol{x}, t),
\end{aligned}
$$

and streaming:

$$
f_{i}\left(\boldsymbol{x}+\boldsymbol{c}_{i}, t+1\right)=f_{i}^{\star}(\boldsymbol{x}, t),
$$

where the superscript $\star$ denotes post-collision quantities here and henceforth. If not otherwise stated, the dependence on the space and the time will be tacitly assumed in the rest of the paper. The term $\mathcal{F}_{i}$ accounts for external body forces $\boldsymbol{F}=\left[F_{x}, F_{y}, F_{z}\right]$ and it will be discussed later. The fluid density $\rho$ and velocity $\boldsymbol{u}=\left[u_{x}, u_{y}, u_{z}\right]$ are computed as ${ }^{73}$

$$
\begin{aligned}
\rho & =\sum_{i} f_{i}, \\
\rho \boldsymbol{u} & =\sum_{i} f_{i} \boldsymbol{c}_{i}+\frac{\boldsymbol{F}}{2},
\end{aligned}
$$

respectively. The second term of the right-hand side of Eq. (3) is the BGK collision operator, that forces all the populations to relax to a discrete local equilibrium $f_{i}^{e q}$ with the same rate $\omega=\left(\nu / c_{s}^{2}+1 / 2\right)^{-1}, \nu$ being the fluid kinematic viscosity. Following the works by Malaspinas ${ }^{68}$ and Coreixas et al. ${ }^{35,37,69}$, the full potential of the D3Q27 lattice can only be achieved by correctly expanding the equilibrium distribution onto the complete basis composed of 27 Hermite polynomials

$$
\begin{aligned}
f_{i}^{e q}= & w_{i} \rho\left\{1+\frac{\boldsymbol{c}_{i} \cdot \boldsymbol{u}}{c_{s}^{2}}+\frac{1}{2 c_{s}^{4}}\left[\mathcal{H}_{i x x}^{(2)} u_{x}^{2}+\mathcal{H}_{i y y}^{(2)} u_{y}^{2}+\mathcal{H}_{i z z}^{(2)} u_{z}^{2}+2\left(\mathcal{H}_{i x y}^{(2)} u_{x} u_{y}+\mathcal{H}_{i x z}^{(2)} u_{x} u_{z}+\mathcal{H}_{i y z}^{(2)} u_{y} u_{z}\right)\right]\right. \\
& +\frac{1}{2 c_{s}^{6}}\left[\mathcal{H}_{i x x y}^{(3)} u_{x}^{2} u_{y}+\mathcal{H}_{i x x z}^{(3)} u_{x}^{2} u_{z}+\mathcal{H}_{i x y y}^{(3)} u_{x} u_{y}^{2}+\mathcal{H}_{i x z z}^{(3)} u_{x} u_{z}^{2}+\mathcal{H}_{i y z z}^{(3)} u_{y} u_{z}^{2}+\mathcal{H}_{i y y z}^{(3)} u_{y}^{2} u_{z}+2 \mathcal{H}_{i x y z}^{(3)} u_{x} u_{y} u_{z}\right] \\
& +\frac{1}{4 c_{s}^{8}}\left[\mathcal{H}_{i x x y y}^{(4)} u_{x}^{2} u_{y}^{2}+\mathcal{H}_{i x x z z}^{(4)} u_{x}^{2} u_{z}^{2}+\mathcal{H}_{i y y z z}^{(4)} u_{y}^{2} u_{z}^{2}+2\left(\mathcal{H}_{i x y z z}^{(4)} u_{x} u_{y} u_{z}^{2}+\mathcal{H}_{i x y y z}^{(4)} u_{x} u_{y}^{2} u_{z}+\mathcal{H}_{i x x y z}^{(4)} u_{x}^{2} u_{y} u_{z}\right)\right] \\
& +\frac{1}{4 c_{s}^{10}}\left[\mathcal{H}_{i x x y z z}^{(5)} u_{x}^{2} u_{y} u_{z}^{2}+\mathcal{H}_{i x x y y z}^{(5)} u_{x}^{2} u_{y}^{2} u_{z}+\mathcal{H}_{i x y y z z}^{(5)} u_{x} u_{y}^{2} u_{z}^{2}\right]
\end{aligned}
$$




$$
\left.+\frac{1}{8 c_{s}^{12}} \mathcal{H}_{i x x y y z z}^{(6)} u_{x}^{2} u_{y}^{2} u_{z}^{2}\right\},
$$

where the weights are $w_{0}=8 / 27, w_{1 \ldots 6}=2 / 27$, $w_{7 \ldots 18}=1 / 54, w_{19 \ldots 26}=1 / 216$ and $c_{s}=1 / \sqrt{3}$ is the lattice sound speed. $\mathcal{H}_{i}^{(n)}$ denotes the $n$ th-order Hermite polynomial tensor. Coefficients before these tensors are $\left(n_{x} ! n_{y} ! n_{z} ! c_{s}^{2 n}\right)^{-1}$, where $n_{x}, n_{y}$ and $n_{z}$ are the number of occurrences of $x, y$ and $z$ respectively. Full expressions of the Hermite polynomials are compiled in App. A.

Before going any further, it is important to understand that in the present context the sole purpose of the extended equilibrium state in Eq. (7) is to derive Galilean invariant equilibrium and forcing CMs. Hence, only these CMs are required for the implementation of the D3Q27-CM-LBM with or without external forcing. In addition, one can note that Eq. (7) recovers the classical second-order truncated equilibrium if $\mathcal{H}_{i}^{(3)}, \mathcal{H}_{i}^{(4)}, \mathcal{H}_{i}^{(5)}$ and $\mathcal{H}_{i}^{(6)}$ are neglected. In addition, one can show that the better macroscopic behavior recovered by sophisticated collision models such as cumulant ${ }^{42,44-46}$ and recursive regularized ${ }^{35,68,69}$ based LBMs is only due to this extended equilibrium. Hence, it is sufficient to rely on Eq. (7) if one wants to get rid of non-diagonal velocity-dependent errors in the viscous stress tensor, whereas one might want to change the collision model in order to increase the numerical stability ${ }^{37}$.

Regarding the forcing term, in the continuous Boltzmann equation it is expressed as $\mathcal{F}=-\boldsymbol{F} \cdot \boldsymbol{\nabla}_{\boldsymbol{c}} f$, where $\nabla_{c}$ is the derivative with respect to the mesoscopic velocity $\boldsymbol{c}^{12}$. Based on the Chapman-Enskog expansion, the Navier-Stokes-Fourier equations can be recovered if the force term is approximated as $-\boldsymbol{F} \cdot \boldsymbol{\nabla}_{\boldsymbol{c}} f^{e q}$, since its non-equilibrium contribution only impacts high-order physics (Burnett, Super Burnett, etc). Then, it is possible to write an Hermite expansion of this forcing term based on the expansion of the equilibrium state ${ }^{12}$

$$
\begin{aligned}
\mathcal{F} & =-\boldsymbol{F} \cdot \nabla_{\boldsymbol{c}} f^{e q} \\
& =-\boldsymbol{F} \cdot \nabla_{\boldsymbol{c}}\left(w(\boldsymbol{c}) \sum_{n=0}^{\infty} \frac{1}{n !} \boldsymbol{a}_{e q}^{(n)} \cdot \mathcal{H}^{(n)}\right) \\
& =-\boldsymbol{F} \cdot \sum_{n=0}^{\infty} \frac{1}{n !} \boldsymbol{a}_{e q}^{(n)} \cdot \nabla_{\boldsymbol{c}}\left(w(\boldsymbol{c}) \mathcal{H}^{(n)}\right) \\
& =\omega(\boldsymbol{c}) \sum_{n=1}^{\infty} \frac{1}{n !} \rho\left[\boldsymbol{F} \boldsymbol{u}^{(n-1)}\right] \cdot \mathcal{H}^{(n)}
\end{aligned}
$$

where both Rodrigues' formula and $\boldsymbol{a}_{e q}^{(n-1)}=\rho \boldsymbol{u}^{(n-1)}$ have been used ${ }^{68} \cdot \boldsymbol{u}^{(n-1)}$ is the velocity tensor of rank $(n-1)$. The square bracket [•] stands for cyclic permutations, e.g., for $n=3$,

$$
[\boldsymbol{F u u}]=\boldsymbol{F u u}+\boldsymbol{u F u}+\boldsymbol{u} \boldsymbol{u} \boldsymbol{F}
$$

By adopting the extended equilibrium state related to the D3Q27 lattice (see Eq. (7)), the expansion of the complete forcing term reads as

$$
\begin{aligned}
\mathcal{F}_{i}= & w_{i} \rho\left\{\frac{\boldsymbol{F} \cdot \boldsymbol{c}_{i}}{c_{s}^{2}}+\frac{1}{2 c_{s}^{4}}\left[\mathcal{H}_{i x x}^{(2)}\left(2 u_{x} F_{x}\right)+\mathcal{H}_{i y y}^{(2)}\left(2 u_{y} F_{y}\right)+\mathcal{H}_{i z z}^{(2)}\left(2 u_{z} F_{z}\right)+2 \mathcal{H}_{i x y}^{(2)}\left(u_{x} F_{y}+u_{y} F_{x}\right)+2 \mathcal{H}_{i x z}^{(2)}\left(u_{x} F_{z}+u_{z} F_{x}\right)\right.\right. \\
& \left.+2 \mathcal{H}_{i y z}^{(2)}\left(u_{y} F_{z}+u_{z} F_{y}\right)\right]+\frac{1}{2 c_{s}^{6}}\left[\mathcal{H}_{i x x y}^{(3)}\left(2 u_{x} u_{y} F_{x}+u_{x}^{2} F_{y}\right)+\mathcal{H}_{i x x z}^{(3)}\left(2 u_{x} u_{z} F_{x}+u_{x}^{2} F_{z}\right)+\mathcal{H}_{i x y y}^{(3)}\left(2 u_{x} u_{y} F_{y}+u_{y}^{2} F_{x}\right)\right. \\
& +\mathcal{H}_{i x z z}^{(3)}\left(2 u_{x} u_{z} F_{z}+u_{z}^{2} F_{x}\right)+\mathcal{H}_{i y z z}^{(3)}\left(2 u_{y} u_{z} F_{z}+u_{z}^{2} F_{y}\right)+\mathcal{H}_{i y y z}^{(3)}\left(2 u_{y} u_{z} F_{y}+u_{y}^{2} F_{z}\right)+2 \mathcal{H}_{i x y z}^{(3)}\left(u_{x} u_{y} F_{z}+u_{x} F_{y} u_{z}\right. \\
& \left.\left.+F_{x} u_{y} u_{z}\right)\right]+\frac{1}{4 c_{s}^{8}}\left[\mathcal{H}_{i x x y y}^{(4)}\left(2 u_{x}^{2} u_{y} F_{y}+2 u_{x} u_{y}^{2} F_{x}\right)+\mathcal{H}_{i x x z z}^{(4)}\left(2 u_{x}^{2} u_{z} F_{z}+2 u_{x} u_{z}^{2} F_{x}\right)+\mathcal{H}_{i y y z z}^{(4)}\left(2 u_{y}^{2} u_{z} F_{z}+2 u_{y} u_{z}^{2} F_{y}\right)\right. \\
& +2 \mathcal{H}_{i x y z z}^{(4)}\left(2 u_{x} u_{y} u_{z} F_{z}+u_{x} F_{y} u_{z}^{2}+F_{x} u_{y} u_{z}^{2}\right)+2 \mathcal{H}_{i x y y z}^{(4)}\left(2 u_{x} u_{y} u_{z} F_{y}+u_{x} u_{y}^{2} F_{z}+F_{x} u_{y}^{2} u_{z}\right) \\
& \left.+2 \mathcal{H}_{i x x y z}^{(4)}\left(2 u_{x} u_{y} u_{z} F_{x}+u_{y} u_{x}^{2} F_{z}+u_{x}^{2} u_{z} F_{y}\right)\right]+\frac{1}{4 c_{s}^{10}}\left[\mathcal{H}_{i x x y y z}^{(5)}\left(2 u_{x} u_{y}^{2} u_{z} F_{x}+2 u_{x}^{2} u_{y} u_{z} F_{y}+u_{x}^{2} u_{y}^{2} F_{z}\right)\right. \\
& \left.+\mathcal{H}_{i x x y z z}^{(5)}\left(2 u_{x} u_{y} u_{z}^{2} F_{x}+u_{x}^{2} u_{z}^{2} F_{y}+2 u_{x}^{2} u_{y} u_{z} F_{z}\right)+\mathcal{H}_{i x y y z z}^{(5)}\left(u_{y}^{2} u_{z}^{2} F_{x}+2 u_{x} u_{y} u_{z}^{2} F_{y}+2 u_{x} u_{y}^{2} u_{z} F_{z}\right)\right] \\
& \left.+\frac{1}{8 c_{s}^{12}} \mathcal{H}_{i x x y y z z}^{(6)}\left(2 u_{x} u_{y}^{2} u_{z}^{2} F_{x}+2 u_{x}^{2} u_{y} u_{z}^{2} F_{y}+2 u_{x}^{2} u_{y}^{2} u_{z} F_{z}\right)\right\}
\end{aligned}
$$

where $1 / c_{s}^{2 n}$ terms appear due to the use of discrete Hermite polynomials $\mathcal{H}_{i}^{(n)}$. Once again, the full form in Eq. (9) is only used to derive Galilean invariant $\mathrm{CMs}$ of the forcing term. As a consequence, only these CMs are required for the implementation of the D3Q27-CM-LBM with external forcing, while the extended equilibrium state only serves a theoretical purpose in the present context. Indeed, by following the methodology proposed by Coreixas et al. ${ }^{37}$, it is possible to extend the above forcing term to any collision model, and this is illustrated in App. E for the RM-based 
MRT collision model. Regarding the forcing term itself, one can notice that the popular formula by Guo et al. ${ }^{73}$ is recovered if terms proportional to $\mathcal{H}_{i}^{(3)}, \mathcal{H}_{i}^{(4)}, \mathcal{H}_{i}^{(5)}$ and $\mathcal{H}_{i}^{(6)}$ are neglected.

Now, let us focus on central moments. The pillar to design any CMs-based collision operator is to shift the lattice directions by the local fluid velocity ${ }^{36}$. Therefore, it is possible to define $\overline{\boldsymbol{c}}_{i}=\left[\left|\bar{c}_{i x}\right\rangle,\left|\bar{c}_{i y}\right\rangle,\left|\bar{c}_{i z}\right\rangle\right]$, where

$$
\begin{aligned}
\left|\bar{c}_{i x}\right\rangle & =\left|c_{i x}-u_{x}\right\rangle, \\
\left|\bar{c}_{i y}\right\rangle & =\left|c_{i y}-u_{y}\right\rangle, \\
\left|\bar{c}_{i z}\right\rangle & =\left|c_{i z}-u_{z}\right\rangle .
\end{aligned}
$$

One possibility is to adopt the basis proposed in Ref. ${ }^{61}$, which is $\overline{\mathcal{T}}=\left[\bar{T}_{0}, \ldots, \bar{T}_{i}, \ldots, \bar{T}_{26}\right]$ with

$$
\begin{aligned}
& \left|\bar{T}_{0}\right\rangle=|1, \ldots, 1\rangle, \\
& \left|\bar{T}_{1}\right\rangle=\left|\bar{c}_{i x}\right\rangle \text {, } \\
& \left|\bar{T}_{2}\right\rangle=\left|\bar{c}_{i y}\right\rangle \text {, } \\
& \left|\bar{T}_{3}\right\rangle=\left|\bar{c}_{i z}\right\rangle \text {, } \\
& \left|\bar{T}_{4}\right\rangle=\left|\bar{c}_{i x} \bar{c}_{i y}\right\rangle, \\
& \left|\bar{T}_{5}\right\rangle=\left|\bar{c}_{i x} \bar{c}_{i z}\right\rangle, \\
& \left|\bar{T}_{6}\right\rangle=\left|\bar{c}_{i y} \bar{c}_{i z}\right\rangle, \\
& \left|\bar{T}_{7}\right\rangle=\left|\bar{c}_{i x}^{2}-\bar{c}_{i y}^{2}\right\rangle, \\
& \left|\bar{T}_{8}\right\rangle=\left|\bar{c}_{i x}^{2}-\bar{c}_{i z}^{2}\right\rangle, \\
& \left|\bar{T}_{9}\right\rangle=\left|\bar{c}_{i x}^{2}+\bar{c}_{i y}^{2}+\bar{c}_{i z}^{2}\right\rangle, \\
& \left|\bar{T}_{10}\right\rangle=\left|\bar{c}_{i x} \bar{c}_{i y}^{2}+\bar{c}_{i x} \bar{c}_{i z}^{2}\right\rangle, \\
& \left|\bar{T}_{11}\right\rangle=\left|\bar{c}_{i x}^{2} \bar{c}_{i y}+\bar{c}_{i y} \bar{c}_{i z}^{2}\right\rangle, \\
& \left|\bar{T}_{12}\right\rangle=\left|\bar{c}_{i x}^{2} \bar{c}_{i z}+\bar{c}_{i y}^{2} \bar{c}_{i z}\right\rangle, \\
& \left|\bar{T}_{13}\right\rangle=\left|\bar{c}_{i x} \bar{c}_{i y}^{2}-\bar{c}_{i x} \bar{c}_{i z}^{2}\right\rangle, \\
& \left|\bar{T}_{14}\right\rangle=\left|\bar{c}_{i x}^{2} \bar{c}_{i y}-\bar{c}_{i y} \bar{c}_{i z}^{2}\right\rangle \text {, } \\
& \left|\bar{T}_{15}\right\rangle=\left|\bar{c}_{i x}^{2} \bar{c}_{i z}-\bar{c}_{i y}^{2} \bar{c}_{i z}\right\rangle, \\
& \left|\bar{T}_{16}\right\rangle=\left|\bar{c}_{i x} \bar{c}_{i y} \bar{c}_{i z}\right\rangle, \\
& \left|\bar{T}_{17}\right\rangle=\left|\bar{c}_{i x}^{2} \bar{c}_{i y}^{2}+\bar{c}_{i x}^{2} \bar{c}_{i z}^{2}+\bar{c}_{i y}^{2} \bar{c}_{i z}^{2}\right\rangle, \\
& \left|\bar{T}_{18}\right\rangle=\left|\bar{c}_{i x}^{2} \bar{c}_{i y}^{2}+\bar{c}_{i x}^{2} \bar{c}_{i z}^{2}-\bar{c}_{i y}^{2} \bar{c}_{i z}^{2}\right\rangle, \\
& \left|\bar{T}_{19}\right\rangle=\left|\bar{c}_{i x}^{2} \bar{c}_{i y}^{2}-\bar{c}_{i x}^{2} \bar{c}_{i z}^{2}\right\rangle \text {, } \\
& \left|\bar{T}_{20}\right\rangle=\left|\bar{c}_{i x}^{2} \bar{c}_{i y} \bar{c}_{i z}\right\rangle, \\
& \left|\bar{T}_{21}\right\rangle=\left|\bar{c}_{i x} \bar{c}_{i y}^{2} \bar{c}_{i z}\right\rangle \text {, } \\
& \left|\bar{T}_{22}\right\rangle=\left|\bar{c}_{i x} \bar{c}_{i y} \bar{c}_{i z}^{2}\right\rangle \text {, } \\
& \left|\bar{T}_{23}\right\rangle=\left|\bar{c}_{i x} \bar{c}_{i y}^{2} \bar{c}_{i z}^{2}\right\rangle \text {, } \\
& \left|\bar{T}_{24}\right\rangle=\left|\bar{c}_{i x}^{2} \bar{c}_{i y} \bar{c}_{i z}^{2}\right\rangle \text {, } \\
& \left|\bar{T}_{25}\right\rangle=\left|\bar{c}_{i x}^{2} \bar{c}_{i y}^{2} \bar{c}_{i z}\right\rangle \text {, } \\
& \left|\bar{T}_{26}\right\rangle=\left|\bar{c}_{i x}^{2} \bar{c}_{i y}^{2} \bar{c}_{i z}^{2}\right\rangle \text {. }
\end{aligned}
$$

Let us first recall important results concerning the force-free CM-LBM. Pre-collision, equilibrium and post-collision CMs are defined as

$$
\begin{aligned}
\left|k_{i}\right\rangle & =\left[k_{0}, \ldots, k_{i}, \ldots, k_{26}\right]^{\top}, \\
\left|k_{i}^{e q}\right\rangle & =\left[k_{0}^{e q}, \ldots, k_{i}^{e q}, \ldots, k_{26}^{e q}\right]^{\top}, \\
\left|k_{i}^{\star}\right\rangle & =\left[k_{0}^{\star}, \ldots, k_{i}^{\star}, \ldots, k_{26}^{\star}\right]^{\top},
\end{aligned}
$$

respectively. The first two quantities are evaluated by applying the transformation matrix $\overline{\mathcal{T}}$ to the corresponding distributions, i.e,

$$
\begin{aligned}
\left|k_{i}\right\rangle & =\overline{\mathcal{T}}^{\top}\left|f_{i}\right\rangle, \\
\left|k_{i}^{e q}\right\rangle & =\overline{\mathcal{T}}^{\top}\left|f_{i}^{e q}\right\rangle,
\end{aligned}
$$

where $\left|f_{i}^{e q}\right\rangle=\left[f_{0}^{e q}, \ldots f_{i}^{e q}, \ldots f_{26}^{e q}\right]^{\top}$. By adopting a Hermite polynomial expansion of the equilibrium state up to $\mathcal{H}_{i}^{(6)}$ in Eq. (7), equilibrium CMs read as follows

$$
\begin{aligned}
& k_{0}^{e q}=\rho, \\
& k_{9}^{e q}=3 \rho c_{s}^{2}, \\
& k_{17}^{e q}=\rho c_{s}^{2}, \\
& k_{18}^{e q}=\rho c_{s}^{4}, \\
& k_{26}^{e q}=\rho c_{s}^{6},
\end{aligned}
$$

with $k_{1 \ldots 8}^{e q}=k_{10 \ldots 16}^{e q}=k_{19 \ldots 25}^{e q}=0$. Notably, only five equilibrium CMs assume non-zero values, and they have the same form as those of the continuous Maxwellian. In other words, Galilean invariant equilibrium CMs are naturally obtained when the full set of Hermite polynomials is considered, as originally demonstrated in Ref. ${ }^{70}$. This is in agreement with the fact that all collision models are based on the extended equilibrium in Eq. (7) when high-order velocity terms are not neglected. Hence, all collision models should recover the same level of Galilean invariance, even if their corresponding equilibrium moments might depend on velocity terms, which might have an impact on their numerical stability ${ }^{37}$.

The post-collision CMs can be written as

$$
\left|k_{i}^{\star}\right\rangle=(\mathbf{I}-\boldsymbol{\Lambda}) \overline{\mathcal{T}}^{\top}\left|f_{i}\right\rangle+\boldsymbol{\Lambda} \overline{\mathcal{T}}^{\top}\left|f_{i}^{e q}\right\rangle
$$

or

$$
\left|k_{i}^{\star}\right\rangle=(\mathbf{I}-\boldsymbol{\Lambda})\left|k_{i}\right\rangle+\boldsymbol{\Lambda}\left|k_{i}^{e q}\right\rangle,
$$

where $\mathbf{I}$ is the $27 \times 27$ unit tensor and

$$
\boldsymbol{\Lambda}=\operatorname{diag}[1,1,1,1, \omega, \omega, \omega, \omega, \omega, 1, \ldots, 1]
$$

is the $27 \times 27$ relaxation matrix adopted in the present work. The latter allows us to increase the numerical stability through (1) the overdissipation of acoustic waves ${ }^{77}$, and (2) the equilibration of high-order moments ${ }^{30,78}$. The post-collision state of CMs then becomes

$$
\begin{aligned}
k_{0}^{\star} & =\rho, \\
k_{4}^{\star} & =(1-\omega) k_{4}, \\
k_{5}^{\star} & =(1-\omega) k_{5}, \\
k_{6}^{\star} & =(1-\omega) k_{6} \\
k_{7}^{\star} & =(1-\omega) k_{7}, \\
k_{8}^{\star} & =(1-\omega) k_{8}, \\
k_{9}^{\star} & =3 \rho c_{s}^{2}, \\
k_{17}^{\star} & =\rho c_{s}^{2}, \\
k_{18}^{\star} & =\rho c_{s}^{4}, \\
k_{26}^{\star} & =\rho c_{s}^{6},
\end{aligned}
$$

where

$$
\begin{aligned}
& k_{4}=\sum_{i} f_{i} \bar{c}_{i x} \bar{c}_{i y}, \\
& k_{5}=\sum_{i} f_{i} \bar{c}_{i x} \bar{c}_{i z}
\end{aligned}
$$




$$
\begin{aligned}
& k_{6}=\sum_{i} f_{i} \bar{c}_{i y} \bar{c}_{i z}, \\
& k_{7}=\sum_{i} f_{i}\left(\bar{c}_{i x}^{2}-\bar{c}_{i y}^{2}\right), \\
& k_{8}=\sum_{i} f_{i}\left(\bar{c}_{i x}^{2}-\bar{c}_{i z}^{2}\right),
\end{aligned}
$$

and $k_{1 \ldots 3}^{\star}=k_{10 \ldots 16}^{\star}=k_{19 \ldots 25}^{\star}=0$. Eventually, the post-collision populations are reconstructed as

$$
\left|f_{i}^{\star}\right\rangle=\left(\overline{\mathcal{T}}^{\top}\right)^{-1}\left|k_{i}^{\star}\right\rangle
$$

with $\left|f_{i}^{\star}\right\rangle=\left[f_{0}^{\star}, \ldots f_{i}^{\star}, \ldots f_{26}^{\star}\right]^{\top}$, before being streamed (see Eq. (4)). One can notice that the computation of only five pre-collision CMs and ten post-collision CMs is required in the present work. Hence, not only the two previous choices (equilibrium in Eq. (7) and relaxation matrix in Eq. (17)) lead to a robust and accurate $\mathrm{CM}-\mathrm{LBM}^{70}$, but they also lead to a very concise formulation.

In presence of an external force, the post-collision CMs can be rewritten as:

$$
\left|k_{i}^{\star}\right\rangle=(\mathbf{I}-\boldsymbol{\Lambda}) \overline{\mathcal{T}}^{\top}\left|f_{i}\right\rangle+\boldsymbol{\Lambda} \overline{\mathcal{T}}^{\top}\left|f_{i}^{e q}\right\rangle+\left(\mathbf{I}-\frac{\boldsymbol{\Lambda}}{2}\right) \overline{\mathcal{T}}^{\top}\left|\mathcal{F}_{i}\right\rangle,
$$

or

$$
\left|k_{i}^{\star}\right\rangle=(\mathbf{I}-\boldsymbol{\Lambda})\left|k_{i}\right\rangle+\boldsymbol{\Lambda}\left|k_{i}^{e q}\right\rangle+\left(\mathbf{I}-\frac{\boldsymbol{\Lambda}}{2}\right)\left|R_{i}\right\rangle,
$$

where the CMs of the forcing term are

$$
\left|R_{i}\right\rangle=\overline{\mathcal{T}}^{\top}\left|\mathcal{F}_{i}\right\rangle
$$

Now, let us proceed by assuming the presence of Hermite polynomials of progressively higher order. If only $\mathcal{H}_{i}^{(1)}$ and $\mathcal{H}_{i}^{(2)}$ are considered, Eq. (8) leads to the following CMs for the forcing term:

$$
\begin{aligned}
& R_{1}=F_{x}, \\
& R_{2}=F_{y} \text {, } \\
& R_{3}=F_{z} \text {, } \\
& R_{10}=2 F_{x} c_{s}^{2}-F_{x} u_{y}^{2}-2 F_{y} u_{x} u_{y}-F_{x} u_{z}^{2}-2 F_{z} u_{x} u_{z} \text {, } \\
& R_{11}=2 F_{y} c_{s}^{2}-F_{y} u_{x}^{2}-2 F_{x} u_{y} u_{x}-F_{y} u_{z}^{2}-2 F_{z} u_{y} u_{z} \text {, } \\
& R_{12}=2 F_{z} c_{s}^{2}-F_{z} u_{x}^{2}-2 F_{x} u_{z} u_{x}-F_{z} u_{y}^{2}-2 F_{y} u_{z} u_{y} \text {, } \\
& R_{13}=-F_{x} u_{y}^{2}-2 F_{y} u_{x} u_{y}+F_{x} u_{z}^{2}+2 F_{z} u_{x} u_{z} \text {, } \\
& R_{14}=-F_{y} u_{x}^{2}-2 F_{x} u_{y} u_{x}+F_{y} u_{z}^{2}+2 F_{z} u_{y} u_{z}, \\
& R_{15}=-F_{z} u_{x}^{2}-2 F_{x} u_{z} u_{x}+F_{z} u_{y}^{2}+2 F_{y} u_{z} u_{y} \text {, } \\
& R_{16}=-F_{z} u_{x} u_{y}-F_{y} u_{x} u_{z}-F_{x} u_{y} u_{z} \text {, } \\
& R_{17}=4 F_{y} u_{x}^{2} u_{y}+4 F_{z} u_{x}^{2} u_{z}+4 F_{x} u_{x} u_{y}^{2}+4 F_{x} u_{x} u_{z}^{2}+4 F_{z} u_{y}^{2} u_{z}+4 F_{y} u_{y} u_{z}^{2} \text {, } \\
& R_{18}=4 F_{y} u_{x}^{2} u_{y}+4 F_{z} u_{x}^{2} u_{z}+4 F_{x} u_{x} u_{y}^{2}+4 F_{x} u_{x} u_{z}^{2}-4 F_{z} u_{y}^{2} u_{z}-4 F_{y} u_{y} u_{z}^{2} \text {, } \\
& R_{19}=4 u_{x}\left(F_{x} u_{y}^{2}+F_{y} u_{x} u_{y}-F_{x} u_{z}^{2}-F_{z} u_{x} u_{z}\right) \text {, } \\
& R_{20}=2 u_{x}\left(F_{z} u_{x} u_{y}+F_{y} u_{x} u_{z}+2 F_{x} u_{y} u_{z}\right) \text {, } \\
& R_{21}=2 u_{y}\left(F_{z} u_{x} u_{y}+2 F_{y} u_{x} u_{z}+F_{x} u_{y} u_{z}\right) \text {, } \\
& R_{22}=2 u_{z}\left(2 F_{z} u_{x} u_{y}+F_{y} u_{x} u_{z}+F_{x} u_{y} u_{z}\right) \text {, } \\
& R_{23}=F_{x} c_{s}^{4}-3 F_{x} u_{y}^{2} u_{z}^{2}-6 F_{z} u_{x} u_{y}^{2} u_{z}-F_{x} u_{y}^{2} / 3-6 F_{y} u_{x} u_{y} u_{z}^{2}-2 F_{y} u_{x} u_{y} / 3-F_{x} u_{z}^{2} / 3-2 F_{z} u_{x} u_{z} / 3 \text {, } \\
& R_{24}=F_{y} c_{s}^{4}-3 F_{y} u_{x}^{2} u_{z}^{2}-6 F_{z} u_{y} u_{x}^{2} u_{z}-F_{y} u_{x}^{2} / 3-6 F_{x} u_{y} u_{x} u_{z}^{2}-2 F_{x} u_{y} u_{x} / 3-F_{y} u_{z}^{2} / 3-2 F_{z} u_{y} u_{z} / 3 \text {, } \\
& R_{25}=F_{z} c_{s}^{4}-3 F_{z} u_{x}^{2} u_{y}^{2}-6 F_{y} u_{z} u_{x}^{2} u_{y}-F_{z} u_{x}^{2} / 3-6 F_{x} u_{z} u_{x} u_{y}^{2}-2 F_{x} u_{z} u_{x} / 3-F_{z} u_{y}^{2} / 3-2 F_{y} u_{z} u_{y} / 3 \text {, } \\
& R_{26}=8\left(F_{z} u_{x}^{2} u_{y}^{2} u_{z}+F_{y} u_{x}^{2} u_{y} u_{z}^{2}+F_{x} u_{x} u_{y}^{2} u_{z}^{2}\right)+4 c_{s}^{2}\left(F_{y} u_{x}^{2} u_{y}+F_{z} u_{x}^{2} u_{z}+F_{x} u_{x} u_{y}^{2}+F_{x} u_{x} u_{z}^{2}+F_{z} u_{y}^{2} u_{z}+F_{y} u_{y} u_{z}^{2}\right) \text {, }
\end{aligned}
$$

with $R_{0}=R_{4 \ldots 9}=0$. One can notice that most of these CMs are not Galilean invariant since they contain velocity-dependent terms. By further taking into account third-order Hermite polynomials, terms proportional to the square of the velocity vanish, and the corresponding CMs become

$$
\begin{aligned}
R_{1} & =F_{x}, \\
R_{2} & =F_{y}, \\
R_{3} & =F_{z}, \\
R_{10} & =2 F_{x} c_{s}^{2},
\end{aligned}
$$

$$
\begin{aligned}
R_{11}= & 2 F_{y} c_{s}^{2}, \\
R_{12}= & 2 F_{z} c_{s}^{2}, \\
R_{17}= & -2 F_{y} u_{x}^{2} u_{y}-2 F_{z} u_{x}^{2} u_{z}-2 F_{x} u_{x} u_{y}^{2}-2 F_{x} u_{x} u_{z}^{2} \\
& -2 F_{z} u_{y}^{2} u_{z}-2 F_{y} u_{y} u_{z}^{2}, \\
R_{18}= & -2 F_{y} u_{x}^{2} u_{y}-2 F_{z} u_{x}^{2} u_{z}-2 F_{x} u_{x} u_{y}^{2}-2 F_{x} u_{x} u_{z}^{2} \\
& +2 F_{z} u_{y}^{2} u_{z}+2 F_{y} u_{y} u_{z}^{2}, \\
R_{19}= & -2 u_{x}\left(F_{x} u_{y}^{2}+F_{y} u_{x} u_{y}-F_{x} u_{z}^{2}-F_{z} u_{x} u_{z}\right), \\
R_{20}= & -u_{x}\left(F_{z} u_{x} u_{y}+F_{y} u_{x} u_{z}+2 F_{x} u_{y} u_{z}\right),
\end{aligned}
$$




$$
\begin{aligned}
R_{21}= & -u_{y}\left(F_{z} u_{x} u_{y}+2 F_{y} u_{x} u_{z}+F_{x} u_{y} u_{z}\right), \\
R_{22}= & -u_{z}\left(2 F_{z} u_{x} u_{y}+F_{y} u_{x} u_{z}+F_{x} u_{y} u_{z}\right), \\
R_{23}= & F_{x} c_{s}^{4}+3 F_{x} u_{y}^{2} u_{z}^{2}+6 F_{z} u_{x} u_{y}^{2} u_{z}+6 F_{y} u_{x} u_{y} u_{z}^{2}, \\
R_{24}= & F_{y} c_{s}^{4}+3 F_{y} u_{x}^{2} u_{z}^{2}+6 F_{z} u_{y} u_{x}^{2} u_{z}+6 F_{x} u_{y} u_{x} u_{z}^{2}, \\
R_{25}= & F_{z} c_{s}^{4}+3 F_{z} u_{x}^{2} u_{y}^{2}+6 F_{y} u_{z} u_{x}^{2} u_{y}+6 F_{x} u_{z} u_{x} u_{y}^{2}, \\
R_{26}= & -12\left(F_{z} u_{x}^{2} u_{y}^{2} u_{z}-F_{y} u_{x}^{2} u_{y} u_{z}^{2}-F_{x} u_{x} u_{y}^{2} u_{z}^{2}\right) \\
& +2 c_{s}^{2}\left(F_{y} u_{x}^{2} u_{y}-F_{z} u_{x}^{2} u_{z}-F_{x} u_{x} u_{y}^{2}-F_{x} u_{x} u_{z}^{2}\right. \\
& \left.-F_{z} u_{y}^{2} u_{z}-F_{y} u_{y} u_{z}^{2}\right),
\end{aligned}
$$

with now $R_{0}=R_{4 \ldots 9}=R_{13 \ldots 16}=0$. If $\mathcal{H}_{i}^{(4)}$ polynomials are further considered in the definition of the forcing term in Eq. (8), we obtain

$$
\begin{aligned}
R_{1} & =F_{x}, \\
R_{2} & =F_{y}, \\
R_{3} & =F_{z}, \\
R_{10} & =2 F_{x} c_{s}^{2}, \\
R_{11} & =2 F_{y} c_{s}^{2}, \\
R_{12} & =2 F_{z} c_{s}^{2}, \\
R_{23} & =F_{x} c_{s}^{4}-F_{x} u_{y}^{2} u_{z}^{2}-2 F_{z} u_{x} u_{y}^{2} u_{z}-2 F_{y} u_{x} u_{y} u_{z}^{2}, \\
R_{24} & =F_{y} c_{s}^{4}-F_{y} u_{x}^{2} u_{z}^{2}-2 F_{z} u_{y} u_{x}^{2} u_{z}-2 F_{x} u_{y} u_{x} u_{z}^{2}, \\
R_{25} & =F_{z} c_{s}^{4}-F_{z} u_{x}^{2} u_{y}^{2}-2 F_{y} u_{z} u_{x}^{2} u_{y}-2 F_{x} u_{z} u_{x} u_{y}^{2}, \\
R_{26} & =8 u_{x} u_{y} u_{z}\left(F_{z} u_{x} u_{y}+F_{y} u_{x} u_{z}+F_{x} u_{y} u_{z}\right),
\end{aligned}
$$

with $R_{0}=R_{4 \ldots 9}=R_{13 \ldots 22}=0$. By adding $\mathcal{H}_{i}^{(5)}$, CMs are

$$
\begin{aligned}
R_{1} & =F_{x}, \\
R_{2} & =F_{y}, \\
R_{3} & =F_{z}, \\
R_{10} & =2 F_{x} c_{s}^{2}, \\
R_{11} & =2 F_{y} c_{s}^{2}, \\
R_{12} & =2 F_{z} c_{s}^{2}, \\
R_{23} & =F_{x} c_{s}^{4}, \\
R_{24} & =F_{y} c_{s}^{4}, \\
R_{25} & =F_{z} c_{s}^{4}, \\
R_{26} & =-2 u_{x} u_{y} u_{z}\left(F_{z} u_{x} u_{y}+F_{y} u_{x} u_{z}+F_{x} u_{y} u_{z}\right) .
\end{aligned}
$$

Finally by matching the Hermite polynomial expansion of the forcing term to the one of the extended equilibrium state in Eq. (7), the following expressions are derived:

$$
\begin{aligned}
R_{1} & =F_{x}, \\
R_{2} & =F_{y}, \\
R_{3} & =F_{z}, \\
R_{10} & =2 F_{x} c_{s}^{2}, \\
R_{11} & =2 F_{y} c_{s}^{2}, \\
R_{12} & =2 F_{z} c_{s}^{2}, \\
R_{23} & =F_{x} c_{s}^{4}, \\
R_{24} & =F_{y} c_{s}^{4}, \\
R_{25} & =F_{z} c_{s}^{4},
\end{aligned}
$$

with $R_{0}=R_{4 \ldots 9}=R_{13 \ldots 22}=R_{26}=0$, and where all the velocity-dependent terms have vanished, eventually leading to a fully Galilean invariant forcing term.
Before going any further, let us point out some interesting properties of the present approach. First, the same expressions of $\left|\mathcal{R}_{i}\right\rangle$ in Eqs. (28) can be achieved when CMs are computed from the continuous Maxwellian distribution $^{48}$. This is consistent with a recent paper by De Rosis \& Luo ${ }^{70}$, where it is shown that the CMs of the discrete equilibrium distribution reduce to those of the continuous counterpart when the full set of Hermite polynomials is considered. Hence the proposed approach consistently lead to Galilean invariant forcing terms in accordance with the velocity discretization of interest. This methodology can then be extended to any kind of lattices in a straightforward and a priori manner, i.e., without having to rely on the CMs of the Maxwellian equilibrium state. Second, as an alternative to the present Hermite expansion of the forcing term, the latter could have been expressed by the formula $\boldsymbol{F} \cdot\left(\boldsymbol{c}_{i}-\boldsymbol{u}\right) f_{i}^{e q} /\left(\rho c_{s}^{2}\right)$ in He et al. ${ }^{75}$. However, we find that, in this case, the CMs of the force show a dependence on the third power of the velocity. This is due to the fact that the forcing term $-\boldsymbol{F} \cdot \boldsymbol{\nabla}_{\boldsymbol{c}_{i}} f^{e q}$ is only approximated as $-\boldsymbol{F} \cdot \frac{\boldsymbol{c}_{i}-\boldsymbol{u}}{c_{s}^{2}} f^{e q}$ in the pioneering work by He et al. ${ }^{75}$. This is consistent with the analysis presented in ${ }^{76}$ for the D2Q9 space, and such a problem is avoided in the present work thanks to the extended Hermite polynomial expansion.

Eventually, post-collision CMs accounting for sixth-order Hermite polynomials in both equilibrium populations and forcing term are:

$$
\begin{aligned}
k_{0}^{\star} & =\rho, \\
k_{1}^{\star} & =F_{x} / 2, \\
k_{2}^{\star} & =F_{y} / 2, \\
k_{3}^{\star} & =F_{z} / 2, \\
k_{4}^{\star} & =(1-\omega) k_{4}, \\
k_{5}^{\star} & =(1-\omega) k_{5}, \\
k_{6}^{\star} & =(1-\omega) k_{6} \\
k_{7}^{\star} & =(1-\omega) k_{7}, \\
k_{8}^{\star} & =(1-\omega) k_{8}, \\
k_{9}^{\star} & =3 \rho c_{s}^{2}, \\
k_{10}^{\star} & =F_{x} c_{s}^{2}, \\
k_{11}^{\star} & =F_{y} c_{s}^{2}, \\
k_{12}^{\star} & =F_{z} c_{s}^{2}, \\
k_{17}^{\star} & =\rho c_{s}^{2}, \\
k_{18}^{\star} & =\rho c_{s}^{4}, \\
k_{23}^{\star} & =F_{x} c_{s}^{4} / 2, \\
k_{24}^{\star} & =F_{y} c_{s}^{4} / 2, \\
k_{25}^{\star} & =F_{z} c_{s}^{4} / 2, \\
k_{26}^{\star} & =\rho c_{s}^{6},
\end{aligned}
$$

with $k_{13 \ldots 16}^{\star}=k_{19 \ldots 22}^{\star}=0$. Once again, only five pre-collision CMs need to be computed in the present work. Nevertheless, the inclusion of the forcing term now requires the computation of nine more post-collision CMs, and this brings the number of non-zero post-collision CMs to nineteen instead of ten for the force-free case in Eqs. (18). It is important to note that by adopting central-Hermite moments (CHMs) instead of the more conventional CMs, it might even be possible to reduce the number of non-zero terms to nine and six for CHM-LBMs with and without 
forcing term respectively. This is explained by the fact that, the CHM framework is the only one that makes $c_{s}$-dependent terms vanish, while the cumulant based approach still contains some of them ${ }^{37}$. The behavior of CHM-LBMs is currently being investigated, and corresponding results will be presented in a forthcoming paper.

To conclude, a time iteration of the present algorithm can be summarized as follows:

1. Computation of macroscopic variables by Eq. (6);

2. Evaluation of pre-collision CMs $k_{4 \ldots 8}$ by Eq. (19);

3. Calculation of post-collision CMs as in Eq. (29);

4. Reconstruction of post-collision populations by Eq. (20);

5. Streaming (see Eq. (4)).

For the sake of completeness, a script is provided to allow the reader to perform all the symbolic manipulations required for the implementation of the present approach ${ }^{79}$.

\section{NUMERICAL TESTS}

In this Section, the accuracy, stability and universality of the present approach are discussed using several numerical testcases. Unless differently specified, the density field is initialized as $\rho(\boldsymbol{x}, t=0)=\rho_{0}$, with $\rho_{0}=1$, and the fluid is initially at rest, i.e., $\boldsymbol{u}(\boldsymbol{x}, t=0)=0$. Velocity boundary conditions are assigned through the regularized technique $^{80}$. Six test cases of increasing complexity are investigated: (1) four-rolls mill, (2) Hartmann flow, (3) two-, (4) three-dimensional Orszag-Tang vortex, (5) static droplet, and (6) the Rayleigh-Taylor instability.

The first three tests are two-dimensional (i.e, only one point is used for the spatial discretization in the $z$ direction), while the last one is a fully three-dimensional case. In the first scenario, the effect of a force driving the flow is simulated. In the second, third and fourth ones, an electrically conductive fluid is considered and the proposed treatment of the forcing scheme is applied to the Lorentz force. A self-consistent internal force is considered in order to simulate a two-components flow in the fifth scenario. Finally, the flexibility of the present approach to deal with more sophisticated LB models is shown through simulations of the Rayleigh-Taylor instability by means of the color-gradient method.

In all the tests, a diffusive scaling is adopted for the computation of the time step $\Delta t$, i.e., $\Delta t \propto(\Delta x)^{2}$ with $\Delta x$ being the space step. Hence, the focus will be put on the incompressible behavior of the proposed methodology, which is a common approximation for the simulation of low-Mach number flows ${ }^{4}$. In addition, all quantities are expressed in lattice units hereafter.

To further evaluate the robustness and the accuracy of the present method (referred to as $\mathrm{M}_{1}$ ), a comparative study with four other forcing schemes is conducted:

$-\mathrm{M}_{2}$ : a recent effort by Fei \& $\mathrm{Luo}^{47}$, where equilibrium CMs are derived from the continuous Maxwellian distribution (equivalent to the six-order truncated expansion of the discrete equilibrium state), and

\begin{tabular}{c|c|c}
\hline \hline Model & Equilibrium distribution & Forcing treatment \\
\hline $\mathrm{M}_{1}$ & Discrete, 6th order & Full Hermite expansion \\
$\mathrm{M}_{2}$ & Continuous & He et al. ${ }^{75}$ \\
$\mathrm{M}_{3}$ & Discrete, 2 nd order & He et al. ${ }^{75}$ \\
$\mathrm{M}_{4}$ & Discrete, 6th order & Guo et al..$^{73}$ \\
$\mathrm{M}_{5}$ & Discrete, 2nd order & Guo et al. ${ }^{73}$ \\
\hline \hline
\end{tabular}

TABLE I. Synthetic view of the schemes adopted in our comparative study, that differ for the choice of the equilibrium distribution and forcing term treatment.

CMs of the forcing terms are computed from the approximation by He et al. ${ }^{75}$;

$-\mathrm{M}_{3}$ : the methodology discussed in $^{62}$, where only a second-order truncated expansion is used for the discrete equilibrium populations, while the forcing scheme is the same as for $\mathrm{M}_{2}$ (i.e., He et al. ${ }^{75}$ );

$-\mathrm{M}_{4}$ : a model using sixth-order truncated expansion for the discrete equilibrium populations and a second-order force treatment (i.e., Guo et al. ${ }^{73}$ );

$-\mathrm{M}_{5}$ : a model using the second-order truncated expansion for both the discrete equilibrium populations and the forcing term (i.e., Guo et al. ${ }^{73}$ ).

A synthetic overview of the considered models is given in TABLE I.

Comparisons between methods $\mathrm{M}_{1}, \mathrm{M}_{4}$ and $\mathrm{M}_{5}$ are proposed to better understand the contribution of each high-order modification (equilibrium or forcing term) in the context of the Hermite polynomial expansion. $\mathrm{M}_{2}$ and $\mathrm{M}_{4}$, as well as $\mathrm{M}_{3}$ and $\mathrm{M}_{5}$, further highlight the impact of the forcing scheme since they are based on the same equilibrium CMs. On the contrary, comparing $\mathrm{M}_{2}$ and $\mathrm{M}_{3}$ (or $\mathrm{M}_{4}$ and $\mathrm{M}_{5}$ ) gives more information about the influence of equilibrium CMs.

\section{A. Two-dimensional four-rolls mill}

Let us consider a square periodic box of size $[x, y] \in[0$ : $N]^{2}, N$ being the number of points in each spatial direction. This test simulates the effect of four rotating cylinders that are replaced by a constant body force driving the flow. The latter reads as

$$
\boldsymbol{F}(\boldsymbol{x})=2 \nu u_{0} \psi^{2}[\sin (\psi x) \sin (\psi y), \cos (\psi x) \cos (\psi y)] .
$$

This test is known as four-rolls mill ${ }^{81}$, and admits a steady state. When the latter is reached, the following velocity field is recovered

$$
\boldsymbol{u}(\boldsymbol{x})=u_{0}[\sin (\psi x) \sin (\psi y), \cos (\psi x) \cos (\psi y)],
$$

where $u_{0}$ is the mean flow velocity and $\psi=2 \pi / N$. A contour plot of the velocity magnitude is depicted in FIG. 1, exhibiting the pattern that is typical of this kind of flow.

In the present study, a Reynolds number $\operatorname{Re}=u_{0} N / \nu$ of 100 is considered. To assert the accuracy of the present numerical scheme, a convergence analysis is carried out by varying $N$ as $N=[8,16,32,64,128,256,512]$. Let us collect the velocity field from Eq. (31) and the one from our numerical experiments in the vectors $\mathbf{q}_{\text {an }}$ and $\mathbf{q}_{\text {num }}$, 
respectively. By doing so, it is then possible to compute the $L_{2}$-norm of the relative error $\varepsilon$ between present findings and the analytical solution as

$$
\varepsilon=\frac{\left\|\mathbf{q}_{\text {an }}-\mathbf{q}_{\text {num }}\right\|}{\left\|\mathbf{q}_{\text {an }}\right\|} .
$$

Notice that we consider the numerical solution when the steady state is reached. This is achieved when the relative discrepancy in the velocity magnitude between two successive time steps is lower than a certain threshold $\left(10^{-12}\right.$ in this case). As shown in FIG. 2, the expected second-order accuracy property of the present method $\left(\mathrm{M}_{1}\right)$ is successfully recovered, as an excellent convergence rate equal to 1.998 is found. Moreover, all the considered models show the same accuracy. This result can simply be explained by the fact that discrepancies between these models are proportional to powers of the velocity.

By increasing the mean velocity from $u_{0}=0.001$ to 0.1 , these discrepancies can be exerted, as illustrated in FIG. 3. Generally speaking, models with the highest equilibrium $\left(\mathrm{M}_{1}, \mathrm{M}_{2}\right.$ and $\left.\mathrm{M}_{4}\right)$ show the best accuracy. An optimal convergence rate $\sim 2$ is achieved until $N<32$ and then

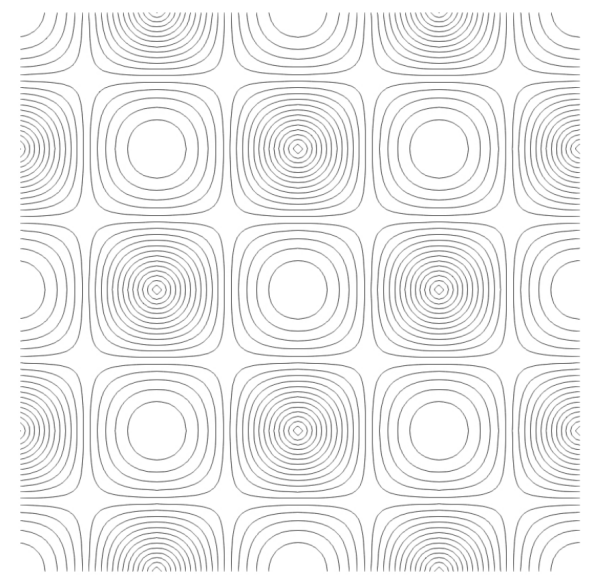

FIG. 1. Four-rolls mill: contour lines of the velocity magnitude. The plot is obtained by generating ten equally spaced intervals ranging from 0 to $u_{0}$.

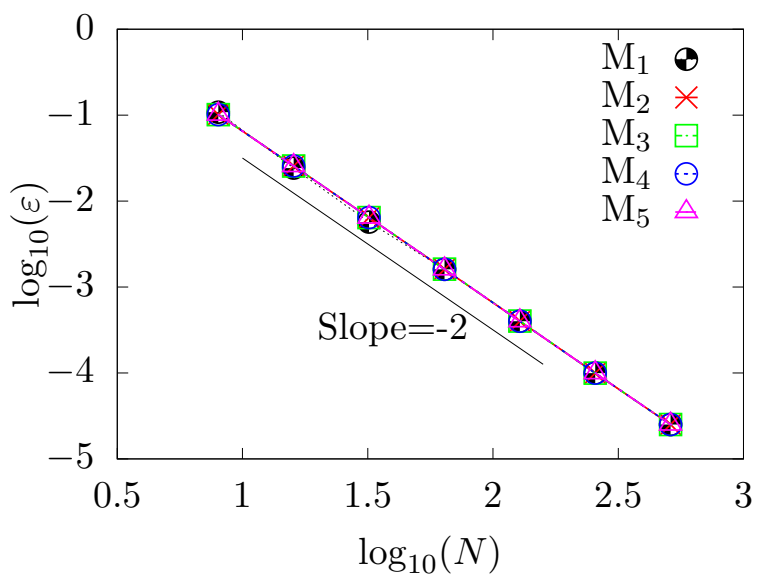

FIG. 2. Four-rolls mill at $u_{0}=0.001$ : results of a convergence analysis by adopting different lattice Boltzmann models. The black continuous line corresponds to the expected second-order accuracy.

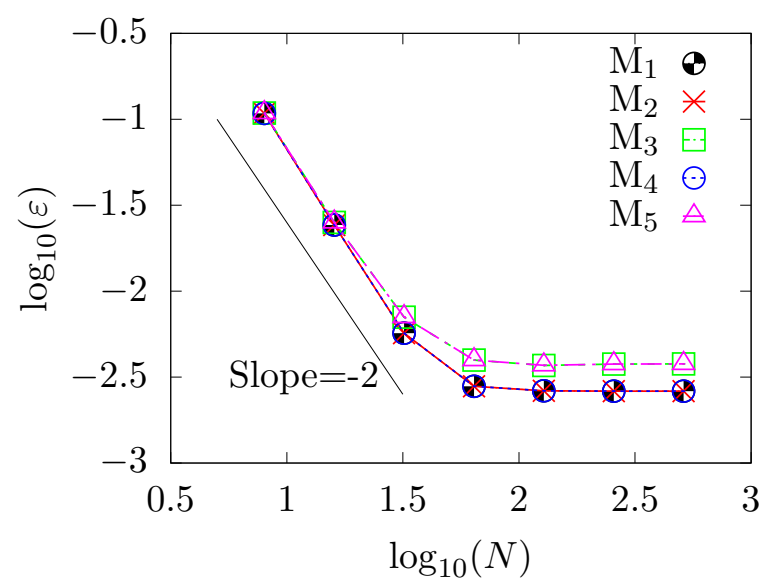

FIG. 3. Four-rolls mill at $u_{0}=0.1$ : results of a convergence analysis by adopting different lattice Boltzmann models. The black continuous line corresponds to the expected second-order accuracy. The detrimental effect played by a poor equilibrium representation affecting $\mathrm{M}_{3}$ and $\mathrm{M}_{5}$ is clearly visible.

curves reach a plateau. Interestingly, $\mathrm{M}_{3}$ and $\mathrm{M}_{5}$ lose the second-order-accurate property of the LBE for a coarser grid resolution, i.e. $N<16$, showing lower accuracy for any larger value of $N$. We address this behavior to the poorer description of the equilibrium distribution. In fact, $\mathrm{M}_{3}$ and $\mathrm{M}_{5}$ are derived by using the classical expression of $f^{e q}$ with Hermite polynomials up to the second order (see TABLE I). These findings are confirmed in TABLE II, where $\log _{10}(\varepsilon)$ is reported for all the models and grid resolutions. Further

\begin{tabular}{cccccc}
\hline \hline$N$ & $\mathrm{M}_{1}$ & $\mathrm{M}_{2}$ & $\mathrm{M}_{3}$ & $\mathrm{M}_{4}$ & $\mathrm{M}_{5}$ \\
\hline 8 & -0.9655 & -0.9655 & -0.9637 & -0.9654 & -0.9636 \\
16 & -1.6153 & -1.6153 & -1.6000 & -1.6152 & -1.5999 \\
32 & -2.2445 & -2.2445 & -2.1513 & -2.2442 & -2.1510 \\
64 & -2.5534 & -2.5534 & -2.4010 & -2.5531 & -2.4007 \\
128 & -2.5797 & -2.5797 & -2.4319 & -2.5795 & -2.4317 \\
256 & -2.5818 & -2.5818 & -2.4244 & -2.5817 & -2.4243 \\
512 & -2.5820 & -2.5820 & -2.4230 & -2.5820 & -2.4230 \\
\hline \hline
\end{tabular}

TABLE II. Four-rolls mill at $u_{0}=0.1$ : base- 10 logarithms of the relative errors for all the models and grid resolutions. $\mathrm{M}_{1}$ and $\mathrm{M}_{2}$ show the best performance.

insights are given in FIG. 4, where results from a convergence analysis at $u_{0}=0.3$ are depicted. Again, the highest accuracy is obtained by adopting $\mathrm{M}_{1}, \mathrm{M}_{2}$ and $\mathrm{M}_{4}$. However, we notice that the overall accuracy tends to deteriorate as the plateau is reached for lower resolutions with all considered models. This can be attributed to velocity-dependent error terms in the viscous stress tensor, which are drastically reduced by adopting the correct equilibrium state ${ }^{37}$. This is further highlighted in FIG. 5, where results are reported for $\mathrm{M}_{1}$ with $u_{0}=0.001,0.1,0.3$.

\section{B. Two-dimensional Hartmann flow}

Let us now consider the flow of an electrically conductive fluid of magnetic resistivity $\eta=\nu$, and which is subjected to a magnetic field $\boldsymbol{b}$. The interest reader shall refer to App. C for further details about the computation of the magnetic 


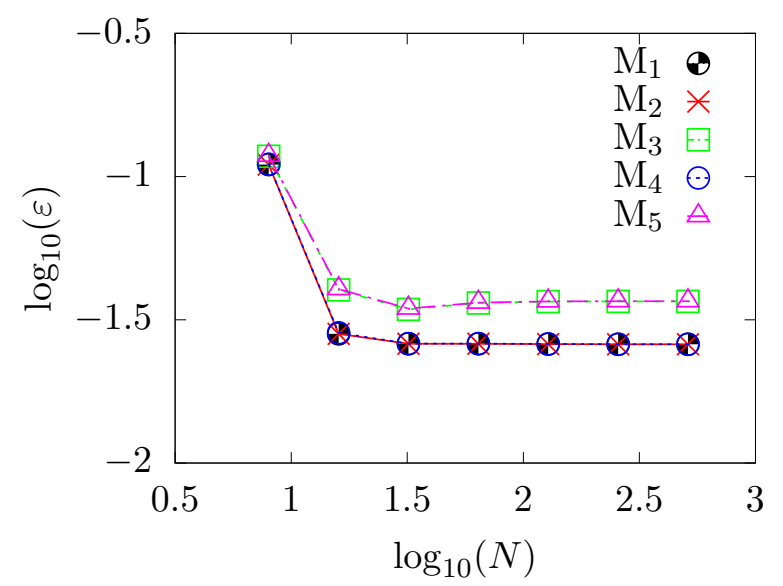

FIG. 4. Four-rolls mill at $u_{0}=0.3$ : results of a convergence analysis by adopting different lattice Boltzmann models. Again, models based on second-order equilibrium $\left(\mathrm{M}_{3}\right.$ and $\left.\mathrm{M}_{5}\right)$ exhibit the poorest accuracy.

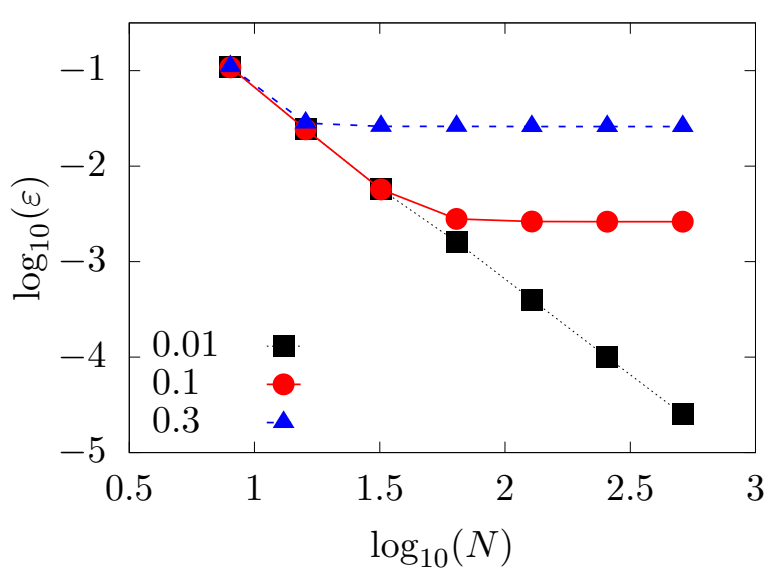

FIG. 5. Four-rolls mill: results of a convergence are reported by adopting $\mathrm{M}_{1}$ at $u_{0}=0.001$ (black squares), 0.1 (red circles) and 0.3 (blue triangles). As $u_{0}$ increases, the convergence and accuracy of the solution deteriorates due to remaining Mach-number errors.

field $\boldsymbol{b}$ in the lattice Boltzmann framework. Let us assume a rectangular domain of length $L_{x}$ and height $L_{y}$. Initial conditions consist of

$$
\boldsymbol{b}(\boldsymbol{x}, t=0)=\left[0, b_{y 0}, 0\right] .
$$

We reproduce the so-called Hartmann flow, that it is analogous to the Poiseuille flow, but in the former case: (1) the fluid is assumed to be conductive, and (2) a constant uniform vertical magnetic field $\left(b_{y 0}\right)$ is enforced at the bottom and top walls, where the no-slip condition is enforced too. At the other sides, the magnetic field is assumed to be periodic. Magnetic boundary conditions are assigned according to ${ }^{82}$. The analytical solution of the Hartmann flow reads as follows:

$$
u_{x}(\boldsymbol{x}, t)=\frac{4 \nu u_{0}}{L_{y} b_{y 0} \tanh (\mathrm{Ha})}\left[1-\frac{\cosh \left(\mathrm{Ha} y^{\prime} / L_{y}\right)}{\cosh (\mathrm{Ha})}\right]
$$

where $y^{\prime}=2 y-L_{y}$ and the Hartmann number is defined as $\mathrm{Ha}=b_{y 0} L_{y} /{\sqrt{4 \rho_{0} \nu \eta}}^{83}$. Moreover, Eq. (33) is prescribed at the inlet section, while an outflow boundary condition is applied at the opposite side of the domain as $\boldsymbol{n}^{\ddagger} \cdot \nabla \boldsymbol{u}=0, \boldsymbol{n}^{\ddagger}$ being the unit vector normal to the boundary. The presence of the magnetic field gives rise to the Lorentz force $\boldsymbol{F}=\boldsymbol{j} \times \boldsymbol{b}$, $j$ being the electric current that is computed directly from the populations ${ }^{84}$.

In FIG. 6, results obtained with the present approach are compared to the analytical solution in Eq. (33) for four values of the Hartmann number, i.e., $\mathrm{Ha}=1,3,10,20$. From them, it is clear that $\mathrm{M}_{1}$ is able to correctly recover the physics of the Hartmann flow when the grid mesh is fine enough. The impact of the grid mesh, as well as, the convergence order of the present method is further studied by evaluating the relative error by Eq. (32) for different values of $L_{y} \in[5: 1025]$, and using the analytical solution in Eq. (33). In FIG. 7, the relative error is plotted against the number of points required to discretize the vertical dimension. Interestingly, a poor convergence is experienced for low values of $L_{y}$, especially for high values of Ha. This behavior can be explained by the presence of progressively thinner Hartmann layers, the latter requiring a larger number of grid points to be successfully and accurately captured.

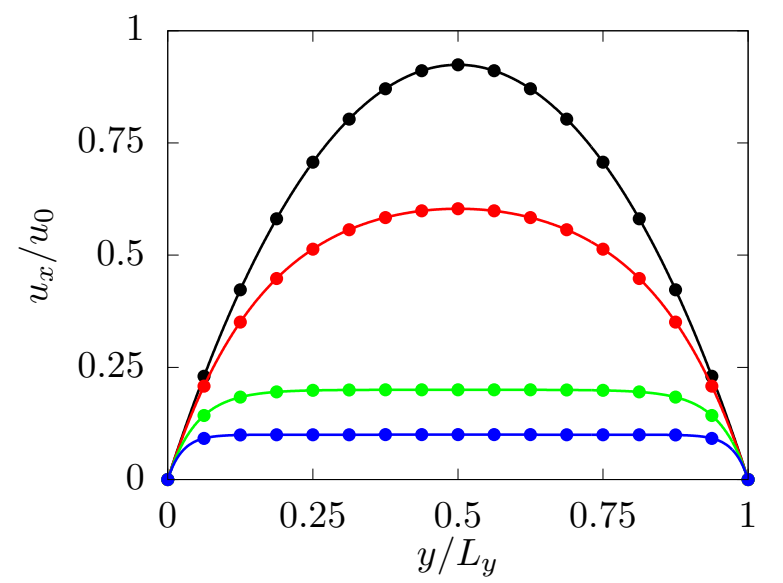

FIG. 6. Hartmann flow: normalized axial velocity at $\mathrm{Ha}=1$ (black), 3 (red), 10 (green) and 20 (blue). Continuous lines and circles respectively account for the analytical predictions and the numerical solutions obtained with $\mathrm{M}_{1}$ using $L_{y}=1025$.

In addition, this test was further considered to compare the behavior of the proposed approach with other forcing schemes. Since the Hartmann flow is a purely one-dimensional problem (i.e., $u_{y}=u_{z}=0$ ), then only terms proportional to $u_{x}$ remain in Eqs. (7) and (9). As a consequence, it is expected that $\mathrm{M}_{1}$ and $\mathrm{M}_{4}$ reduce to $M_{5}$, whereas $M_{2}$ should recover the behavior of $M_{3}$. Hence, for any combination of $\mathrm{Ha}$ and $L_{y}$, it has been confirmed that high-order approaches lead exactly to the same results, in terms of relative errors and velocity profiles, as their low-order versions.

\section{Two-dimensional Orszag-Tang vortex}

The next test case consists in the simulation of the Orszag-Tang vortex problem ${ }^{83,85}$. Its simulation represents a hard task since smaller and smaller structures appear in 


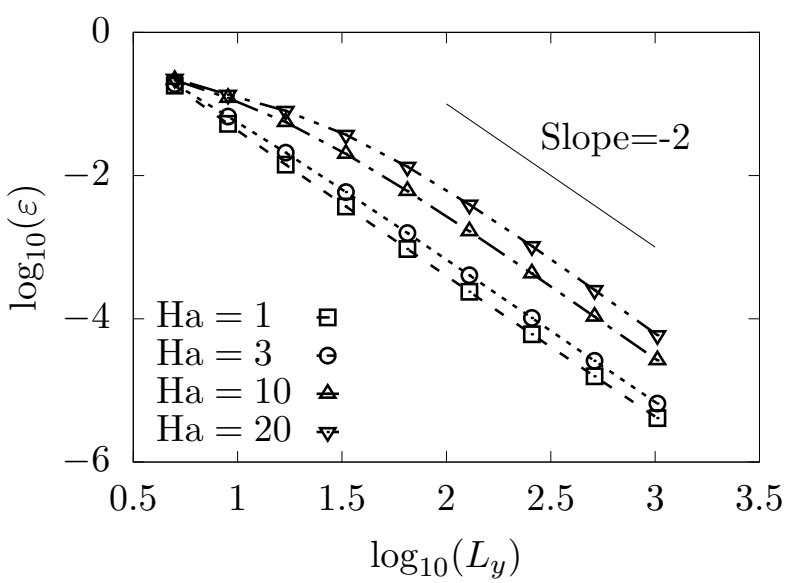

FIG. 7. Hartmann flow simulation using $\mathrm{M}_{1}$ : convergence analysis for $\mathrm{Ha}=1$ (squares, dashed line), 3 (circles, dotted line), 10 (triangles, dot-dashed line) and 20 (inverted triangles, dot-dot-dashed line). The continuous line denotes a convergence rate equal to 2 .

the domain as the Reynolds number grows. This test case is then a perfect candidate to assess (1) the accuracy of the present method to capture the existence of fine flow features, as well as (2) its ability to deal with high local gradients that arise during the simulation ${ }^{66}$.

In what follows, a square periodic box of length $2 \pi$ is considered with a Reynolds number of $\operatorname{Re}=1600$, and initial conditions are set to

$$
\begin{aligned}
\boldsymbol{u}(\boldsymbol{x}, t=0) & =u_{0}[-\sin (\psi y), \sin (\psi x)], \\
\boldsymbol{b}(\boldsymbol{x}, t=0) & =b_{0}[-\sin (\psi y), \sin (2 \psi x)]
\end{aligned}
$$

In FIG. 8, the mean kinetic energy $\left\langle u^{2}\right\rangle$, normalized by its initial value $\left\langle u_{0}^{2}\right\rangle$ with $\langle\bullet\rangle$ standing for the average over the whole domain, is reported for different values of the Mach number, i.e., Ma $=u_{0} / c_{s}=[0.07,0.14,0.28]$ with $c_{s}=1 / \sqrt{3}$. Moreover, it is set $b_{0}=u_{0}$. Experiments are carried out by adopting Hermite polynomials up to the second- and sixth-orders for both equilibrium populations and forcing term, corresponding to the models $\mathrm{M}_{1}$ and $\mathrm{M}_{5}$, respectively. Here, the stability properties of the proposed algorithm clearly manifests. In fact, the two strategies are both stable for $\mathrm{Ma}=0.07$ and 0.14 . By further increasing the Mach number, $\mathrm{M}_{5}$ becomes unstable whereas the present approach, $\mathrm{M}_{1}$, leads to the correct behavior.

We also use this scenario to evaluate the accuracy of different LB approaches. In TABLE III, the peak values of the current $j_{\max }$ and vorticity $\zeta_{\max }$ are compared to reference high-resolution spectral values in $^{83}$. All the models show very good accuracy, with the relative errors lower than $0.22 \%$. To further evaluate their robustness, the Mach number is increased up to $\mathrm{Ma}=0.28$ for all the involved forcing schemes. From this, it is found that only $\mathrm{M}_{5}$ manifests stability issues.

Now that the good numerical properties of our approach have been rigorously demonstrated in the $2 \mathrm{D}$ case, its validation will further be carried out considering 3D flows. Due to the non-negligible CPU time required to run these simulations, $\mathrm{M}_{1}$ will only be compared to the second-order formulations $\mathrm{M}_{4}$ and $\mathrm{M}_{5}$, with the exception of the

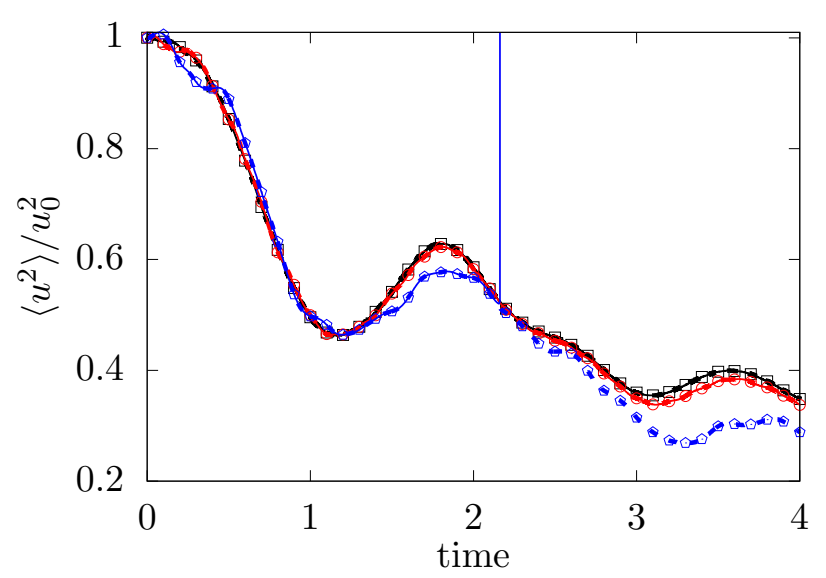

FIG. 8. Orszag-Tang vortex in two dimensions: time history of the kinetic energy normalized by its initial value for different Mach numbers, i.e., $\mathrm{Ma}=0.07$ (black), 0.14 (red) and 0.28 (blue). Continuous and dashed lines account for simulations carried out by using Hermite polynomials (used for both equilibrium populations and forcing term) up to the second- $\left(\mathrm{M}_{5}\right)$ and sixth$\left(\mathrm{M}_{1}\right)$ orders, respectively. The fluid domain consists of 256 points in each direction. The accuracy of these results is assessed by repeating the experiments with a finer grid $\left(512^{2}\right.$ lattice points) and by depicting the corresponding solution with symbols (squares for $\mathrm{Ma}=0.07$, circles for $\mathrm{Ma}=0.14$ and pentagons for $\mathrm{Ma}=0.28)$. From them, it is clear that a mesh convergence has been achieved.

Rayleigh-Taylor instability test case, where data are already available in the literature.

\section{Three-dimensional Orszag-Tang vortex}

This test involves an Orszag-Tang vortex that develops in a three-dimensional cubic periodic box of length $2 \pi$ with the initial conditions defined according to ${ }^{86}$, i.e.,

$$
\begin{aligned}
\boldsymbol{u}(\boldsymbol{x}, t=0)= & u_{0}[2 \sin (y), 2 \sin (x), 0], \\
\boldsymbol{b}(\boldsymbol{x}, t=0)= & 0.8 b_{0}[-2 \sin (2 y)+\sin (z), \\
& 2 \sin (x)+\sin (z), \sin (x)+\sin (y)],
\end{aligned}
$$

with $u_{0}=b_{0}=0.0203$. Similarly to the two-dimensional case, here the flow is characterized by the presence of intermittent small-scale structures. However, the topology of the reconnection of the magnetic field is considerably more complicated in three dimensions ${ }^{87}$. Firstly, the accuracy of the method is assessed by running a simulation at $R e=100$ with three different grid sizes, i.e., $N=[32,64,128]$. In FIG. 9, the time evolution of the peak value of the current magnitude $\left(j_{\max }\right)$ is depicted for the different grid dimensions. As $N$ increases, the numerical solution gets progressively closer to the reference one obtained from a high-resolution pseudo-spectral simulation ${ }^{61}$. This is further quantified by defining the vectors $\mathbf{q}_{\text {ref }}$ and $\mathbf{q}_{\text {num }}$, gathering findings from the reference pseudo-spectral analysis and our results, respectively. Then, we introduce $\varepsilon_{\infty}$ as the $L_{\infty}$-norm of the relative discrepancy, i.e.,

$$
\varepsilon_{\infty}=\frac{\max \left|\mathbf{q}_{\mathrm{ref}}-\mathbf{q}_{\mathrm{num}}\right|}{\max \left|\mathbf{q}_{\mathrm{ref}}\right|,}
$$




\begin{tabular}{cccccccc}
\hline \hline & $t$ & {$[83]$} & $\mathrm{M}_{1}$ & $\mathrm{M}_{2}$ & $\mathrm{M}_{3}$ & $\mathrm{M}_{4}$ & $\mathrm{M}_{5}$ \\
\hline \multirow{2}{*}{$j_{\max }$} & 0.5 & 18.24 & 18.26 & 18.26 & 18.26 & 18.26 & 18.26 \\
& 1 & 46.59 & 46.66 & 46.66 & 46.66 & 46.66 & 46.66 \\
\hline \multirow{2}{*}{$\zeta_{\max }$} & 0.5 & 6.758 & 6.756 & 6.756 & 6.756 & 6.756 & 6.756 \\
& 1 & 14.20 & 14.10 & 14.10 & 14.11 & 14.10 & 14.10 \\
\hline \hline
\end{tabular}

TABLE III. Orszag-Tang vortex: findings from different LB schemes and reference spectral values from Ref. [83] in terms of peak value of the current, $j_{\max }$, and vorticity, $\zeta_{\max }$, at two representative time instants. Reproduced with permission from J. Comput. Phys. 179 (2002). Copyright 2002 Elsevier.

that is of order $10^{-2}$ for the finest grid, thus highlighting the accuracy of the method. Then, in order to evaluate the stability of the algorithm, higher values of the Reynolds number are considered, i.e., $\operatorname{Re}=[570,1040,3040,5600]$. In FIG. (10), the time evolution of $j_{\max }$ is reported for all these Reynolds numbers. From this, it is clear that the present approach $\left(\mathrm{M}_{1}\right)$ remains stable even for the highest value of the Reynolds number. To further highlight the robustness of $\mathrm{M}_{1}$, the case $\mathrm{Re}=570$ (the one that is expected to be the least prone to the onset of instability) is also simulated using this time the BGK collision operator ${ }^{84}$. One can notice that, for the latter collision model, the run blows-up at $t \sim 0.43$. Conversely, the adoption of $\mathrm{CMs}$ (either the sixth- or the second-order one, i.e. $\mathrm{M}_{1}, \mathrm{M}_{4}$ and $\mathrm{M}_{5}$ ) overcome this problem, allowing us to simulate a larger time span for the whole desired set of Re. This is consistent with the observations in $^{66}$ regarding two-dimensional magnetohydrodynamic flows simulated by a CMs-based D2Q9 model. Nonetheless, by increasing the Mach number, $\mathrm{M}_{5}$ becomes unstable as it was already the case in Sec. III C. Finally, a contour plot of the current magnitude is sketched in FIG. (11) at different time instants for the configuration $\mathrm{Re}=570$ with $N=128$. One can appreciate that the present method is able to capture the existence of fine flow features, as well as the presence of strong gradients.

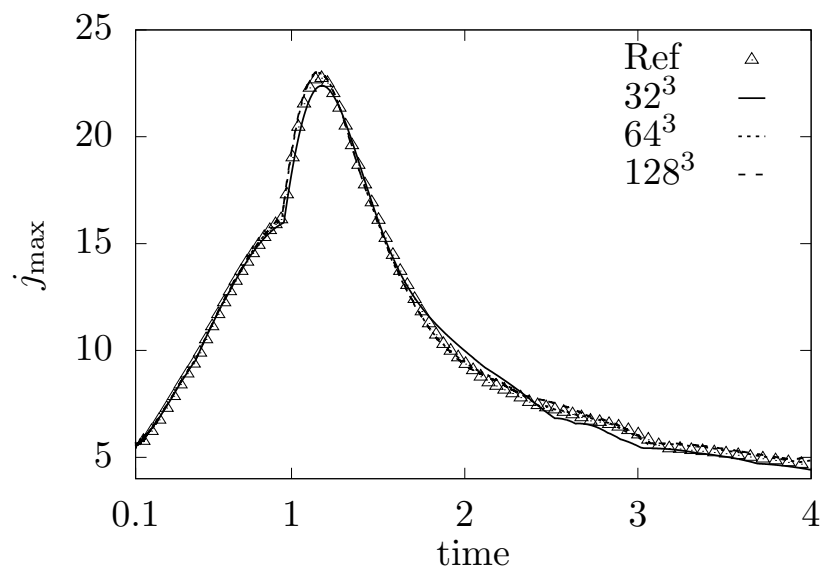

FIG. 9. Orszag-Tang vortex in three dimensions: the time evolutions of the peak value of the current magnitude, $j_{\max }$, for different grid dimensions, i.e., $32^{3}$ (continuous line), $64^{3}$ (dotted line), $128^{3}$ (dashed line), generated by the present method are compared to those from a high-resolution pseudo-spectral simulation from ${ }^{61}$ (triangles, Ref). Reproduced with permission from Phys. Rev. E 95, 013310 (2017). Copyright 2017 American Physical Society.

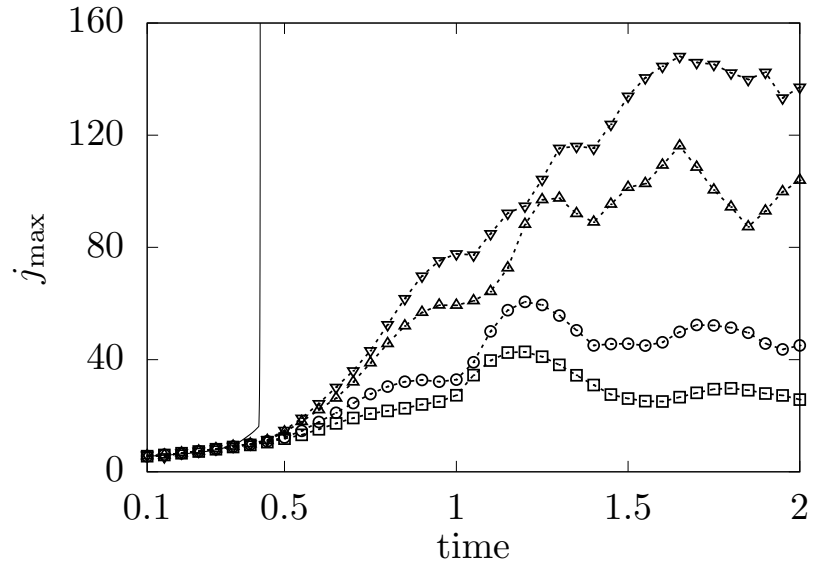

FIG. 10. Orszag-Tang vortex in three dimensions: time evolution of the peak value of the magnitude of the current for different Reynolds numbers. Symbols denote results from simulations by $\mathrm{CMs}$ at $\mathrm{Re}=570$ (squares), $\mathrm{Re}=1040$ (circles), $\mathrm{Re}=3040$ (triangles) and $\mathrm{Re}=5600$ (inverted triangles). The BGK run at $\operatorname{Re}=570$ (continuous line) shows an unstable behavior, while CMs-runs always lead to a stable solution. Every run is conducted with $N=128$.

\section{E. Static droplet}

In the following, the behavior of the present model is further investigated in the context of a multiphase flow simulated through the popular Shan-Chen model ${ }^{88}$. Specifically, the latter introduces an interaction force

$$
\boldsymbol{F}(\boldsymbol{x}, t)=-G \psi(\boldsymbol{x}, t) \sum_{i} w_{i} \boldsymbol{c}_{i} \psi\left(\boldsymbol{x}+\boldsymbol{c}_{i}, t\right),
$$

that mimics the molecular interactions leading to phase segregation. $\psi=1-e^{-\rho}$ is the so-called pseudo-potential and $G$ is a parameter controlling the strength of the interaction.

Let us consider a periodic domain consisting of 200 lattice points in each direction. A droplet of radius $R$ and density equal to $\rho_{0}$ is placed in the center of the domain, while the density is set to $\rho_{0} / 8$ elsewhere. The parameter $G$ is set equal to -6 . The pseudo-potential LBM is known to be prone to numerical instability due to the presence of spurious velocity currents. In FIG. 12, the time evolution of the mean kinetic energy is reported for numerical experiments carried out by using the models $\mathrm{M}_{1}, \mathrm{M}_{4}$ and $\mathrm{M}_{5}$. Here, the beneficial effect of using sixth-order Hermite polynomials for the forcing term are clearly visible. Indeed, the run corresponding to the poorest model (i.e., $\mathrm{M}_{5}$ ) blows-up after $\sim 120$ time iterations. Conversely, the adoption of sixth-order Hermite polynomials allows us to simulate a considerably larger time span, with the kinetic energy that 

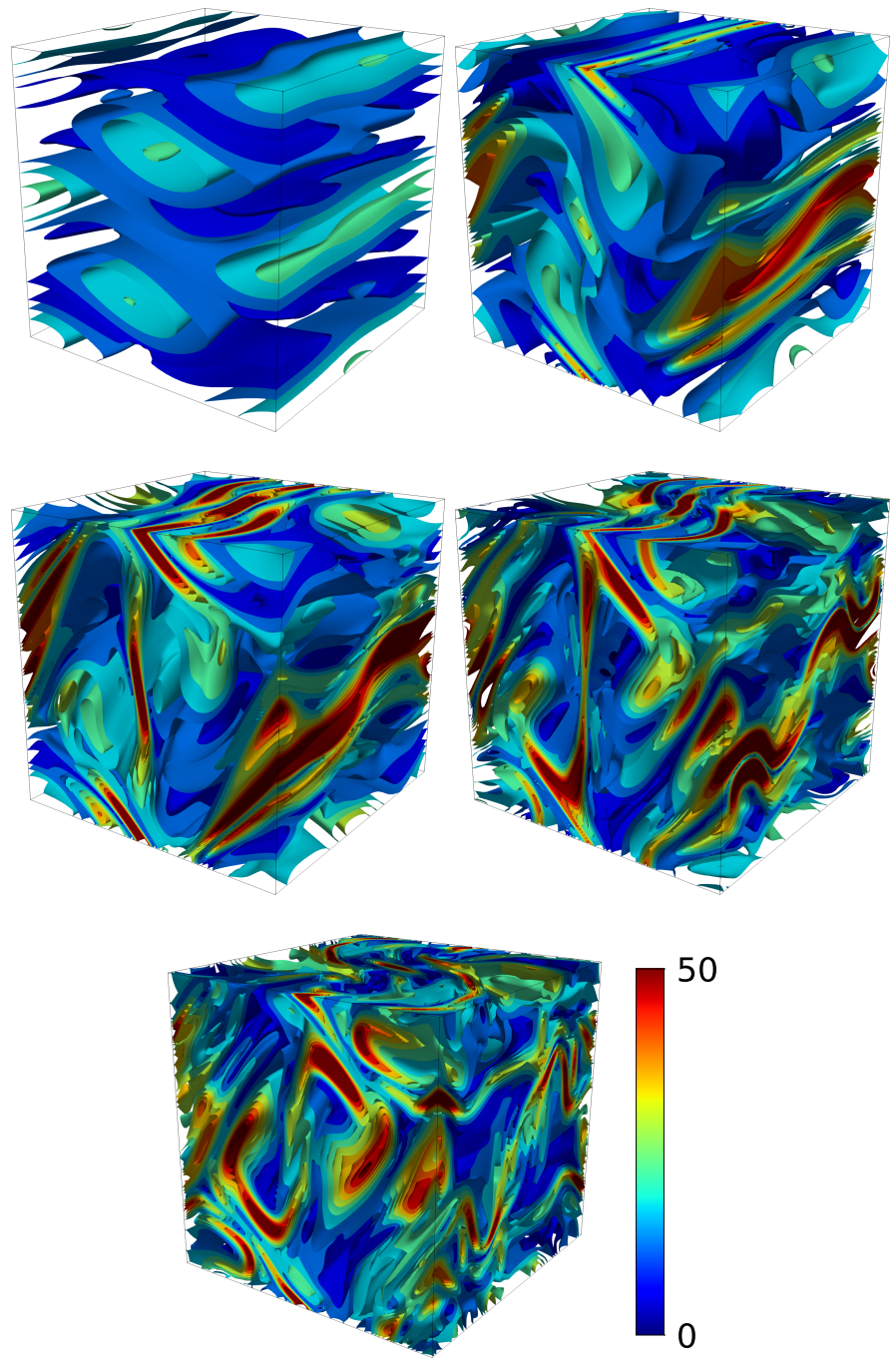

FIG. 11. Simulation of the Orszag-Tang vortex in three dimensions using $\mathrm{M}_{1}$ : time evolution of the (isocontours of the) current magnitude for $\operatorname{Re}=570$ and $N=128$. From top left to bottom, $t=0.1,0.5,1.0,1.5$ and 2.0.

tends to vanish after initial large-amplitude oscillations. This behavior is slightly impacted by the form of the forcing term, as both $\mathrm{M}_{1}$ and $\mathrm{M}_{4}$ lead to stable simulations. A small mismatch between the two forcing schemes is found in the early stage of the simulation, where larger velocity magnitude are encountered. They emphasize the impact of higher-order Hermite polynomials on the forcing term, and suggest a slightly better behavior of $\mathrm{M}_{1}$, as compared to $\mathrm{M}_{4}$, since the former leads to a lower maximal kinetic energy. This mismatch is supposed to be even greater for droplets moving at a speed $u \neq 0$.

In order to compare the computational cost of different approaches, TABLE IV reports the CPU time needed to perform the typical time step normalized by the fastest one. Each $\mathrm{C}++$ serial program runs on a desktop machine equipped by an Intel(R) Core(TM) i7-6700 CPU-3.40 GHz with $16 \mathrm{MB}$ of L3 cache and $32 \mathrm{~GB}$ of RAM. The operating system is CANONICAL UBUNTU 16.10. Very interestingly, the proposed approach exhibits an advantage with respect to the remaining three-dimensional methods that we explain as follows. The real bottleneck of CM-LBMs is represented by the reconstruction of post-collision populations by Eq. (20).

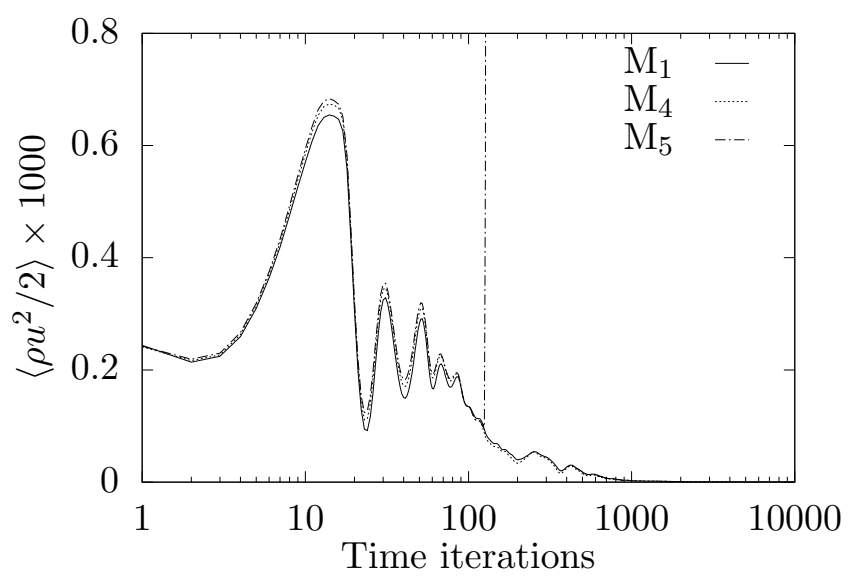

FIG. 12. Static droplet: time evolution of the mean kinetic energy by adopting Hermite polynomials up to the second- (solid line) and sixth- (dotted line) orders.

In this regard, several choices are available. For example, one can be represented by a hand-made implementation of the solution of this linear system by a Cholesky decomposition (the matrix is always positive definite), followed by a backor forward-substitution. A smarter choice could imply the adoption of hardware-optimized numerical libraries (e.g., PARDISO $^{89}$, SuiteSparse ${ }^{90}$, Spooles $\left.{ }^{91}\right)$. However, here we prefer to operate a priori a symbolic solution of the system (see Matlab script in the Supplementary Material). By doing so, we can use the fact that many terms in the vector collecting the post-collision CMs $k_{i}^{\star}$ are zero, thus involving many zero terms in the resultant expressions of $f_{i}^{\star}$ that can be directly neglected in our $\mathrm{C}++$ programs. The very good behavior summarized in TABLE IV stems from the fact that the present model $\mathrm{M}_{1}$ is the one with the highest number of zero terms in $k_{i}^{\star}$, implying the smallest number of floating-point operations to reconstruct $f_{i}^{\star}$. The possibility of using a parallel implementation (by OpenMP or MPI) does not impact the ratios of mutual performance, that is affected only by the number of floating point operations required by each single lattice point. Consequently, even if the present approach does not show a drastic improvement in terms of accuracy, it leads to the shortest wall-clock time one can expect from CM-LBMs with external forcing.

\begin{tabular}{c|c|c}
\hline \hline $\mathrm{M}_{1}$ & $\mathrm{M}_{4}$ & $\mathrm{M}_{5}$ \\
\hline 1 & 1.2 & 1.6 \\
\hline \hline
\end{tabular}

TABLE IV. Static droplet: normalized CPU time needed to perform the typical time step by different three-dimensional models.

\section{F. Rayleigh-Taylor instability}

To further investigate the numerical properties of the present approach, the simulation of the Rayleigh-Taylor instability is carried out hereafter. The fundamental mechanism, upon which this kind of instability relies, can be summarized as follows. Let us consider a heavy fluid on top of a lighter one, both being immiscible fluids that are 
separated by an interface. Assuming that they are subject to a gravitational field, their interface will not be able to stay in its equilibrium state. As a consequence, plumes (spikes) will start forming and flowing downward (upward).

This Rayleigh-Taylor instability mechanism is simulated hereafter by adopting the color-gradient method ${ }^{92-94}$. Recently, Saito et al. ${ }^{67}$ have proposed a CM-based color-gradient model by using third- and second-order terms for the equilibrium and forcing, respectively. Here, the approach is recasted within the present framework, hence adopting sixth-order Hermite polynomials for both the equilibrium and the forcing term. This problem, as well as its solution procedure by the color-gradient method, further shows the universality of the present approach for the simulation of multiphysics flows. The CM-based color-gradient method is briefly outlined in App. D.

Let us consider a three-dimensional domain of size $W \times$ $4 W \times W$, with $W=64$. A fluid of density $\rho_{h}$ is placed over a lighter one of density $\rho_{l}=1$. The initial flow field is perturbed as

$$
\begin{aligned}
& \rho(\boldsymbol{x}, 0)=\rho_{h}, \text { if } y>2 W+0.05 W[\cos (2 \pi x)+\cos (2 \pi z)], \\
& \rho(\boldsymbol{x}, 0)=\rho_{l}, \text { otherwise. }
\end{aligned}
$$

No-slip walls are enforced at the top and bottom sections, while periodic boundaries are assumed at the other sides of the domain. A gravitational body force is considered as

$$
\boldsymbol{F}=-\left[\rho(\boldsymbol{x}, t)-\frac{\rho_{h}+\rho_{l}}{2}\right] \boldsymbol{g},
$$

with $\boldsymbol{g}=(0,-g, 0)$, and $g$ chosen so that $\sqrt{g W}=0.04^{92}$. The problem is governed by two dimensionless parameters: the Reynolds number

$$
\operatorname{Re}=\frac{W \sqrt{g W}}{\nu},
$$

and the Atwood number

$$
\text { At }=\frac{\rho_{h}-\rho_{l}}{\rho_{h}+\rho_{l}},
$$

that are set here to $\mathrm{Re}=512$ and At $=0.5$, respectively. In addition, the characteristic time used for the time evolution of the Rayleigh-Taylor instability is defined as

$$
t_{0}=\frac{t}{\sqrt{W g}} .
$$

In the following, let us denote the bottom and top points of the interface as spike and bubble, respectively. In FIG. 13, the evolution of the interface between the two fluids is sketched at salient time instants. They show that the edge of the spike rolls up at $t / t_{0}=3$. This is consistent with another LB study ${ }^{95}$, where the Rayleigh-Taylor instability has been investigated by means of the multiple-relaxation-time kernel.

More quantitative results, regarding the time evolution of the position of the two reference points, are reported in TABLE V, and depicted in FIG. 14. Present findings are further compared to several models to assess its accuracy: (1) a MRT LB study ${ }^{95}$, (2) a BGK LB model for multiphase flows $^{96},(3)$ a phase-field MRT LB scheme ${ }^{97}$ and (4) a solution of the coupled Navier-Stokes-Cahn-Hilliard equations ${ }^{98}$. From this, it is clear that the present method shows a pretty good agreement with data from the literature. This confirms the good numerical properties of the proposed approach, as well as, its universality.
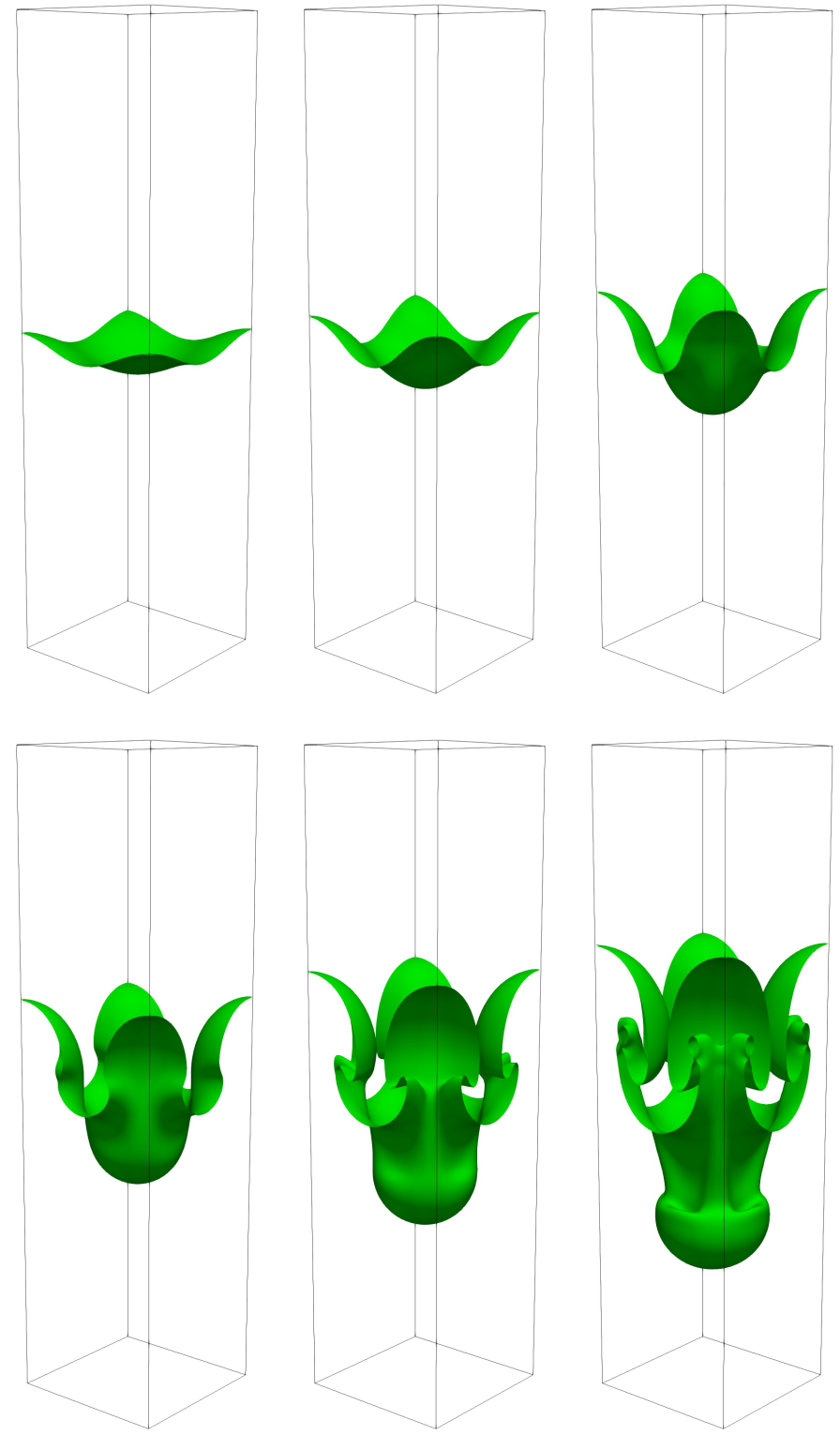

FIG. 13. Rayleigh-Taylor instability: interface evolution at salient time instants, i.e., $t / t_{0}=0.5$ (top left), 1.0 (top center), 1.5 (top right), 2.0 (bottom left), 2.5 (bottom center) and 3.0 (bottom right).

\section{CONCLUSIONS}

In the present work, a systematic way to derive CM-LBMs with external forcing has been proposed for the simulation of multiphysics phenomena. It naturally flows from a previous work based on the D3Q27 velocity discretization ${ }^{70}$, where Galilean invariant CMs were derived from the discrete equilibrium state instead of the continuous Maxwell Boltzmann distribution. Here, by relying on the very same set of 27 Hermite polynomials, Galilean invariant $\mathrm{CMs}$ of the forcing term are also obtained but without any assumption on these CMs. By further equilibrating acoustic and high-order CMs, the present method only requires the computation of five pre-collision CMs. Both points eventually lead to a very concise algorithm.

Interestingly, it is also shown that the present method 


\begin{tabular}{|c|c|c|c|c|c|c|c|c|c|c|}
\hline \multirow[b]{2}{*}{$t / t_{0}$} & \multicolumn{5}{|c|}{ Spike } & \multicolumn{5}{|c|}{ Bubble } \\
\hline & Present & [95] & [96] & [97] & [98] & Present & [95] & [96] & [97] & [98] \\
\hline 0.0 & 1.897 & 1.895 & 1.887 & 1.888 & 1.904 & 2.100 & 2.095 & 2.092 & 2.096 & 2.101 \\
\hline 0.5 & 1.845 & 1.864 & 1.839 & 1.860 & 1.869 & 2.101 & 2.131 & 2.113 & 2.129 & 2.131 \\
\hline 1.0 & 1.753 & 1.763 & 1.744 & 1.755 & 1.776 & 2.202 & 2.230 & 2.229 & 2.228 & 2.224 \\
\hline 1.5 & 1.591 & 1.587 & 1.555 & 1.569 & 1.618 & 2.345 & 2.377 & 2.372 & 2.364 & 2.372 \\
\hline 2.0 & 1.378 & 1.357 & 1.312 & 1.325 & 1.396 & 2.516 & 2.535 & 2.545 & 2.524 & 2.535 \\
\hline 2.5 & 1.121 & 1.085 & 1.022 & 1.037 & 1.149 & 2.682 & 2.695 & 2.693 & 2.672 & 2.688 \\
\hline 3.0 & 0.791 & 0.788 & 0.712 & 0.740 & 0.863 & 2.834 & 2.847 & 2.846 & 2.824 & 2.856 \\
\hline 3.5 & 0.537 & 0.481 & 0.390 & 0.419 & 0.572 & 2.997 & 2.999 & 3.009 & 2.984 & 3.009 \\
\hline 4.0 & 0.233 & 0.160 & 0.060 & 0.090 & 0.271 & 3.184 & 3.179 & 3.178 & 3.164 & 3.181 \\
\hline
\end{tabular}

TABLE V. Rayleigh-Taylor instability: time evolution of the position of the spike and the bubble of the interface at salient time instants. Present results are compared those from (i) a MRT LB study ${ }^{95}$, (ii) a BGK LB model for multiphase flows ${ }^{96}$, (iii) a phase-field MRT LB scheme $^{97}$, and (iv) a solution of the coupled Navier-Stokes-Cahn-Hilliard equations ${ }^{98}$. Reproduced with permission from Phys. Rev. E 96, 013317 (2017). Copyright 2017 American Physical Society. Reproduced from X. He, R. Zhang, S. Chen, and G. D. Doolen, On the three-dimensional Rayleigh-Taylor instability, Phys. Fluids 11, 1143-1152 (1999), with the permission of AIP Publishing. Reproduced with permission from J. Comput. Sci. 17 (2016). Copyright 2016 Elsevier. Reproduced with permission from Comput. Math. Appl. 66 (2013). Copyright 2013 Elsevier.

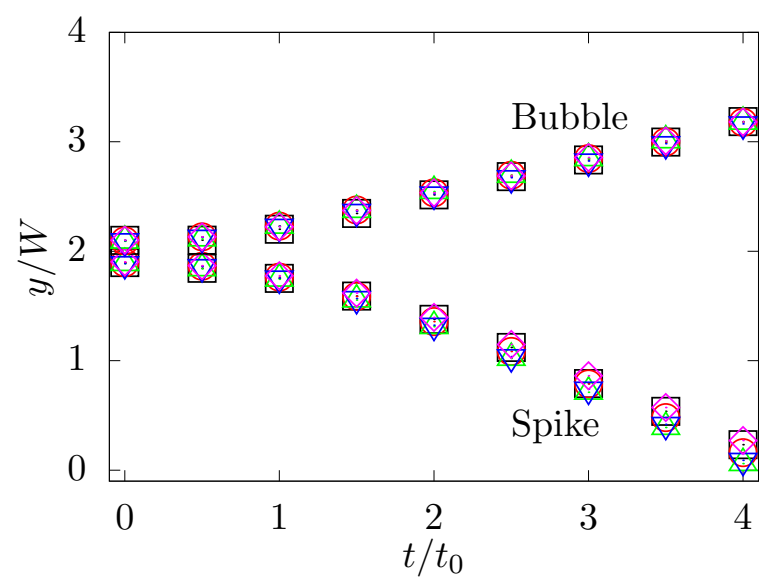

FIG. 14. Rayleigh-Taylor instability: time evolution of the position of the spike and the bubble of the interface at salient time instants. Present results (black squares) are plotted together with those from (i) a MRT LB study ${ }^{95}$ (red circles), (ii) a BGK LB model for multiphase flows ${ }^{96}$ (green triangles), (iii) a phase-field MRT LB scheme ${ }^{97}$ (blue inverted triangles), and (iv) a solution of the coupled Navier-Stokes-Cahn-Hilliard equations ${ }^{98}$ (magenta diamonds). Reproduced with permission from Phys. Rev. E 96, 013317 (2017). Copyright 2017 American Physical Society. Reproduced from X. He, R. Zhang, S. Chen, and G. D. Doolen, On the three-dimensional Rayleigh-Taylor instability, Phys. Fluids 11, 1143-1152 (1999), with the permission of AIP Publishing. Reproduced with permission from J. Comput. Sci. 17 (2016). Copyright 2016 Elsevier. Reproduced with permission from Comput. Math. Appl. 66 (2013). Copyright 2013 Elsevier.

recovers the behavior of the cascaded $\mathrm{LBM}^{48}$. But differently from the latter, the proposed methodology allows the derivation of forcing terms in an a priori way thanks to the tight link existing between the Hermite polynomial expansion, the family of moments considered, and the lattice of discrete velocities. As a consequence, its extension to any kind of collision models and velocity discretizations can be done in a systematic and straightforward manner by following instructions proposed $\mathrm{in}^{37}$. This is illustrated in App. E in the context of the D3Q27-LBM based on RMs.

The simulation of the four-rolls mill, the Hartmann flow, the two- and three-dimensional Orszag-Tang vortex, a static droplet and the Rayleigh-Taylor instability were conducted to evaluate both the numerical behavior as well as the universality of the present method. By further comparing the latter with four other forcing schemes on several of these testcases, its good accuracy and robustness properties were properly demonstrated.

All in all, the consistency with the cascaded LBM, the compactness of the algorithm as well as its excellent numerical properties lead the present scheme to be a very good candidate to perform multiphysics simulations with (or without) the presence of external forces. Regarding future works, the extension to both high-order lattices (that require high-order terms in the Hermite expansion), to other kinds of collision space (raw, Hermite, central Hermite moment and cumulant) as well as regularized approaches ${ }^{37}$ is currently under investigation. Corresponding results shall be presented in the near future.

\section{ACKNOWLEDGMENTS}

This article is based upon work from COST Action MP1305, supported by COST (European Cooperation in Science and Technology). A.D.R. is grateful to Dr. S. Saito for very valuable hints and discussions related to the setup of the simulations of the Rayleigh-Taylor instability.

\section{Appendix A: Hermite polynomials}

Discrete Hermite polynomials are defined as ${ }^{68,69}$

$$
\mathcal{H}_{i}^{(n)}=\frac{\left(-c_{s}^{2}\right)^{n}}{\omega\left(\boldsymbol{c}_{i}\right)} \nabla_{\boldsymbol{c}_{i}}^{n} \omega\left(\boldsymbol{c}_{i}\right)
$$

with the corresponding Gaussian weight function

$$
w\left(\boldsymbol{c}_{i}\right)=\frac{1}{\left(2 \pi c_{s}^{2}\right)^{D / 2}} \exp \left(-\frac{c_{i}^{2}}{2 c_{s}^{2}}\right)
$$

where $D$ is the number of physical dimensions and $c_{s}$ is the lattice constant. In the 3D case, discrete Hermite tensors can be computed using tensor products of their 1D formulation, 


$$
\begin{aligned}
\mathcal{H}_{i x \ldots x y \ldots y z \ldots z}^{(n x+n y+n z)} & =\frac{\left(-c_{s}^{2}\right)^{n x+n y+n z}}{w\left(\boldsymbol{c}_{i}\right)} \nabla_{\boldsymbol{c}_{i}}^{n x+n y+n z} w\left(\boldsymbol{c}_{i}\right) \\
& =\left[\frac{\left(-c_{s}^{2}\right)^{n x}}{w^{\prime}\left(c_{i x}\right)} \nabla_{c_{i x}}^{n x} w^{\prime}\left(c_{i x}\right)\right]\left[\frac{\left(-c_{s}^{2}\right)^{n y}}{w^{\prime}\left(c_{i y}\right)} \nabla_{c_{i y}}^{n y} w^{\prime}\left(c_{i y}\right)\right]\left[\frac{\left(-c_{s}^{2}\right)^{n z}}{w^{\prime}\left(c_{i z}\right)} \nabla_{c_{i z}}^{n z} w^{\prime}\left(c_{i z}\right)\right] \\
& =\mathcal{H}_{i x \ldots x}^{(n x)} \mathcal{H}_{i y \ldots y}^{(n y)} \mathcal{H}_{i z \ldots z}^{(n z)},
\end{aligned}
$$

where $w^{\prime}$ is the one-dimensional version of the Gaussian weight (see Eq. (A2)), and $(n x, n y, n z)$ are the number of occurrences of indexes $(x, y, z)$. Since the D3Q27 lattice is a tensor product of three D1Q3 lattices, then only 1D Hermite polynomials of degree up to $n=2$ can be used for the construction of their 3D counterparts ${ }^{37}$. This means that the degree of each Hermite polynomial will be at most two per direction. Indeed,

$$
\begin{aligned}
\mathcal{H}_{i}^{(0)} & =1, \\
\mathcal{H}_{i x}^{(1)} & =c_{i x}, \\
\mathcal{H}_{i y}^{(1)} & =c_{i y}, \\
\mathcal{H}_{i z}^{(1)} & =c_{i z}, \\
\mathcal{H}_{i x x}^{(2)} & =c_{i x}^{2}-c_{s}^{2}, \\
\mathcal{H}_{i y y}^{(2)} & =c_{i y}^{2}-c_{s}^{2}, \\
\mathcal{H}_{i z z}^{(2)} & =c_{i z}^{2}-c_{s}^{2}, \\
\mathcal{H}_{i x y}^{(2)} & =\mathcal{H}_{i x}^{(1)} \mathcal{H}_{i y}^{(1)}, \\
\mathcal{H}_{i x z}^{(2)} & =\mathcal{H}_{i x}^{(1)} \mathcal{H}_{i z}^{(1)}, \\
\mathcal{H}_{i y z}^{(2)} & =\mathcal{H}_{i y}^{(1)} \mathcal{H}_{i z}^{(1)}, \\
\mathcal{H}_{i x x y}^{(3)} & =\mathcal{H}_{i x x}^{(2)} \mathcal{H}_{i y}^{(1)}, \\
\mathcal{H}_{i x x z}^{(3)} & =\mathcal{H}_{i x x}^{(2)} \mathcal{H}_{i z}^{(1)}, \\
\mathcal{H}_{i y y z}^{(3)} & =\mathcal{H}_{i y y}^{(2)} \mathcal{H}_{i z}^{(1)}, \\
\mathcal{H}_{i x y y}^{(3)} & =\mathcal{H}_{i x}^{(1)} \mathcal{H}_{i y y}^{(2)}, \\
\mathcal{H}_{i x z z}^{(3)} & =\mathcal{H}_{i x}^{(1)} \mathcal{H}_{i z z}^{(2)}, \\
\mathcal{H}_{i y z z}^{(3)} & =\mathcal{H}_{i y}^{(1)} \mathcal{H}_{i z z}^{(2)},
\end{aligned}
$$

$$
\begin{aligned}
\mathcal{H}_{i x y z}^{(3)} & =\mathcal{H}_{i x}^{(1)} \mathcal{H}_{i y}^{(1)} \mathcal{H}_{i z}^{(1)}, \\
\mathcal{H}_{i x x y y}^{(4)} & =\mathcal{H}_{i x x}^{(2)} \mathcal{H}_{i y y}^{(2)}, \\
\mathcal{H}_{i x x z z}^{(4)} & =\mathcal{H}_{i x x}^{(2)} \mathcal{H}_{i z z}^{(2)}, \\
\mathcal{H}_{i y y z z}^{(4)} & =\mathcal{H}_{i y y}^{(2)} \mathcal{H}_{i z z}^{(2)}, \\
\mathcal{H}_{i x x y z}^{(4)} & =\mathcal{H}_{i x x}^{(2)} \mathcal{H}_{i y}^{(1)} \mathcal{H}_{i z}^{(1)}, \\
\mathcal{H}_{i x y y z}^{(4)} & =\mathcal{H}_{i x}^{(1)} \mathcal{H}_{i y y}^{(2)} \mathcal{H}_{i z}^{(1)}, \\
\mathcal{H}_{i x y z z}^{(4)} & =\mathcal{H}_{i x}^{(1)} \mathcal{H}_{i y}^{(1)} \mathcal{H}_{i z z}^{(2)}, \\
\mathcal{H}_{i x x y y z}^{(5)} & =\mathcal{H}_{i x x}^{(2)} \mathcal{H}_{i y y}^{(2)} \mathcal{H}_{i z}^{(1)}, \\
\mathcal{H}_{i x x y z z}^{(5)} & =\mathcal{H}_{i x x}^{(2)} \mathcal{H}_{i y}^{(1)} \mathcal{H}_{i z z}^{(2)}, \\
\mathcal{H}_{i x y y z z}^{(5)} & =\mathcal{H}_{i x}^{(1)} \mathcal{H}_{i y y}^{(2)} \mathcal{H}_{i z z}^{(2)}, \\
\mathcal{H}_{i x x y y z z}^{(6)} & =\mathcal{H}_{i x x}^{(2)} \mathcal{H}_{i y y}^{(2)} \mathcal{H}_{i z z}^{(2)} .
\end{aligned}
$$

\section{Appendix B: Two-dimensional model}

Let us consider an Eulerian basis $\boldsymbol{x}=[x, y]$. Here, we derive the two-dimensional LB model by means of the D2Q9 velocity discretization, where lattice directions $\boldsymbol{c}_{i}=$ $\left[\left|c_{i x}\right\rangle,\left|c_{i y}\right\rangle\right]$ are

$$
\begin{aligned}
& \left|c_{i x}\right\rangle=[0, \quad 1,-1, \quad 0, \quad 0, \quad 1,-1, \quad 1,-1]^{\top},
\end{aligned}
$$

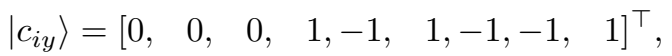

with $i=0 \ldots 8$. Let us define the particle distribution functions $\left|f_{i}\right\rangle=\left[f_{0}, \ldots f_{i}, \ldots f_{8}\right]^{\top}$ and the velocity vector $\boldsymbol{u}=\left[u_{x}, u_{y}\right]$. In two dimensions, the equilibrium distributions $\left|f_{i}^{e q}\right\rangle=\left[f_{0}^{e q}, \ldots f_{i}^{e q}, \ldots f_{8}^{e q}\right]^{\top}$ can be expanded onto a basis of Hermite polynomials $\mathcal{H}^{(n)}$ up to the fourth-order ${ }^{35,68,69}$, i.e.,

$$
f_{i}^{e q}=w_{i} \rho\left(1+\frac{\boldsymbol{c}_{i} \cdot \boldsymbol{u}}{c_{s}^{2}}+\frac{1}{2 c_{s}^{4}} \mathcal{H}_{i}^{(2)}: \boldsymbol{u} \boldsymbol{u}+\frac{1}{2 c_{s}^{6}}\left(\mathcal{H}_{i x x y}^{(3)} u_{x}^{2} u_{y}+\mathcal{H}_{i x y y}^{(3)} u_{x} u_{y}^{2}\right)+\frac{1}{4 c_{s}^{8}} \mathcal{H}_{i x x y y}^{(4)} u_{x}^{2} u_{y}^{2}\right)
$$

where ':' stands for the full contraction of indexes (also known as Frobenius inner product), $w_{0}=4 / 9, w_{1 \ldots 4}=1 / 9$, $w_{5 \ldots 8}=1 / 36$, and $c_{s}=1 / \sqrt{3}$ is the lattice sound speed ${ }^{4}$. Again, the development of a CMs-based collision operator begins by shifting the lattice directions by the local fluid velocity, i.e., $\overline{\boldsymbol{c}}_{i}=\left[\left|\bar{c}_{i x}\right\rangle,\left|\bar{c}_{i y}\right\rangle\right]$, where

$$
\begin{aligned}
\left|\bar{c}_{i x}\right\rangle & =\left|c_{i x}-u_{x}\right\rangle \\
\left|\bar{c}_{i y}\right\rangle & =\left|c_{i y}-u_{y}\right\rangle .
\end{aligned}
$$

Let us choose the basis $\overline{\mathcal{T}}=\left[\bar{T}_{0}, \ldots, \bar{T}_{i}, \ldots, \bar{T}_{8}\right]$ which components are

$$
\begin{aligned}
\left|\bar{T}_{0}\right\rangle & =|1, \ldots, 1\rangle, \\
\left|\bar{T}_{1}\right\rangle & =\left|\bar{c}_{i x}\right\rangle, \\
\left|\bar{T}_{2}\right\rangle & =\left|\bar{c}_{i y}\right\rangle, \\
\left|\bar{T}_{3}\right\rangle & =\left|\bar{c}_{i x}^{2}+\bar{c}_{i y}^{2}\right\rangle, \\
\left|\bar{T}_{4}\right\rangle & =\left|\bar{c}_{i x}^{2}-\bar{c}_{i y}^{2}\right\rangle, \\
\left|\bar{T}_{5}\right\rangle & =\left|\bar{c}_{i x} \bar{c}_{i y}\right\rangle,
\end{aligned}
$$




$$
\begin{aligned}
& \left|\bar{T}_{6}\right\rangle=\left|\bar{c}_{i x}^{2} \bar{c}_{i y}\right\rangle, \\
& \left|\bar{T}_{7}\right\rangle=\left|\bar{c}_{i x} \bar{c}_{i i}^{2}\right\rangle, \\
& \left|\bar{T}_{8}\right\rangle=\left|\bar{c}_{i x}^{2} \bar{c}_{i y}^{2}\right\rangle .
\end{aligned}
$$

Let us collect pre-collision, equilibrium and post-collision CMs as

$$
\begin{aligned}
\left|k_{i}\right\rangle & =\left[k_{0}, \ldots, k_{i}, \ldots, k_{8}\right]^{\top}, \\
\left|k_{i}^{e q}\right\rangle & =\left[k_{0}^{e q}, \ldots, k_{i}^{e q}, \ldots, k_{8}^{e q}\right]^{\top}, \\
\left|k_{i}^{\star}\right\rangle & =\left[k_{0}^{\star}, \ldots, k_{i}^{\star}, \ldots, k_{8}^{\star}\right]^{\top},
\end{aligned}
$$

respectively. The first two quantities are evaluated by applying the transformation matrix $\overline{\mathcal{T}}$ to the corresponding distribution, that is

$$
\begin{aligned}
\left|k_{i}\right\rangle & =\overline{\mathcal{T}}^{\top}\left|f_{i}\right\rangle, \\
\left|k_{i}^{e q}\right\rangle & =\overline{\mathcal{T}}^{\top}\left|f_{i}^{e q}\right\rangle .
\end{aligned}
$$

By adopting equilibrium populations with the full set of Hermite polynomials (see Eq. (B2)), equilibrium CMs are

$$
\begin{aligned}
& k_{0}^{e q}=\rho, \\
& k_{3}^{e q}=2 \rho c_{s}^{2}
\end{aligned}
$$

$$
k_{8}^{e q}=\rho c_{s}^{4} .
$$

and $k_{1,2,4,5,6,7}^{e q}=0$. In analogy with the 3D case, it should be noted that (1) the equilibrium state is fully Galilean invariant since it does not show any dependence on the fluid velocity, and (2) the discrete equilibrium CMs have the same form of the continuous counterpart when the full set of Hermite polynomials is considered.

Again, the post-collision CMs can be written as

$$
\left|k_{i}^{\star}\right\rangle=(\mathbf{I}-\boldsymbol{\Lambda}) \overline{\mathcal{T}}^{\top}\left|f_{i}\right\rangle+\mathbf{\Lambda} \overline{\mathcal{T}}^{\top}\left|f_{i}^{e q}\right\rangle+\left(\mathbf{I}-\frac{\boldsymbol{\Lambda}}{2}\right) \overline{\mathcal{T}}^{\top}\left|\mathcal{F}_{i}\right\rangle
$$

or

$$
\left|k_{i}^{\star}\right\rangle=(\mathbf{I}-\mathbf{\Lambda})\left|k_{i}\right\rangle+\mathbf{\Lambda}\left|k_{i}^{e q}\right\rangle+\left(\mathbf{I}-\frac{\boldsymbol{\Lambda}}{2}\right)\left|R_{i}\right\rangle,
$$

where $\mathbf{I}$ is the $(9 \times 9)$ unit tensor, $\boldsymbol{\Lambda}=$ $\operatorname{diag}[1,1,1,1, \omega, \omega, 1,1,1]$ is a $(9 \times 9)$ relaxation matrix.

Now, let us define the two-dimensional forcing term $\mathcal{F}_{i}$. Specifically, we employ the expression adopted by Huang et $a l .{ }^{76}$ :

$$
\mathcal{F}_{i}=w_{i}\left(\frac{\boldsymbol{F} \cdot \boldsymbol{c}_{i}}{c_{s}} \cdot \mathcal{H}_{i}^{(1)}+\frac{[\boldsymbol{F} \boldsymbol{u}]}{2 c_{s}^{2}} \cdot \mathcal{H}_{i}^{(2)}+\frac{[\boldsymbol{F} \boldsymbol{u u}]}{6 c_{s}^{3}} \cdot \mathcal{H}_{i,[x y y],[x x y]}^{(3)}+\frac{[\boldsymbol{F u u u}]}{24 c_{s}^{4}} \cdot \mathcal{H}_{i,[x x y y]}^{(4)}\right)
$$

where the square bracket in Hermite coefficient denotes permutations. Notice that the popular formula by Guo et al..$^{73}$ is recovered if $\mathcal{H}_{i}^{(3)}$ and $\mathcal{H}_{i}^{(4)}$ are neglected. The $\mathrm{CMs}$ of the forcing term can be computed as

$$
\left|R_{i}\right\rangle=\overline{\mathcal{T}}^{\top}\left|\mathcal{F}_{i}\right\rangle
$$

and read as follows:

$$
\begin{aligned}
& R_{1}=F_{x}, \\
& R_{2}=F_{y}, \\
& R_{6}=F_{y} c_{s}^{2}, \\
& R_{7}=F_{x} c_{s}^{2},
\end{aligned}
$$

and $R_{0,3,4,5,8}=0$. The resultant expressions of the post-collision CMs are:

$$
\begin{aligned}
k_{0}^{\star} & =\rho, \\
k_{1}^{\star} & =F_{x} / 2, \\
k_{2}^{\star} & =F_{y} / 2, \\
k_{3}^{\star} & =2 \rho c_{s}^{2}, \\
k_{4}^{\star} & =(1-\omega) k_{4}, \\
k_{5}^{\star} & =(1-\omega) k_{5}, \\
k_{6}^{\star} & =F_{y} c_{s}^{2} / 2, \\
k_{7}^{\star} & =F_{x} c_{s}^{2} / 2, \\
k_{8}^{\star} & =\rho c_{s}^{4} .
\end{aligned}
$$

Again, one can appreciate that the post-collision state in terms of CMs show simple expressions. Then, the post-collision populations are reconstructed as usual:

$$
\left|f_{i}^{\star}\right\rangle=\left(\overline{\mathcal{T}}^{\top}\right)^{-1}\left|k_{i}^{\star}\right\rangle
$$

with $\left|f_{i}^{\star}\right\rangle=\left[f_{0}^{\star}, \ldots f_{i}^{\star}, \ldots f_{8}^{\star}\right]^{\top}$, that are eventually streamed.

\section{Appendix C: Lattice Boltzmann method for the evolution of the magnetic field}

The evolution of the magnetic field $\boldsymbol{b}$ is predicted by a second LBM based on the D3Q7 velocity space, that is $\forall l \in$ $\llbracket 0,6 \rrbracket$,

$$
\boldsymbol{h}_{l}\left(\boldsymbol{x}+\boldsymbol{\xi}_{l}, t+1\right)=\boldsymbol{h}_{l}(\boldsymbol{x}, t)+\omega_{m}\left[\boldsymbol{h}_{l}^{e q}(\boldsymbol{x}, t)-\boldsymbol{h}_{l}(\boldsymbol{x}, t)\right] .
$$

with $\omega_{m}$ the relaxation frequency that is related to the magnetic resistivity as $\eta=c_{s}^{2}\left(1 / \omega_{m}-1 / 2\right)$, where $c_{s}=1 / 2$ for the D3Q7 lattice. For this particular lattice, discrete velocities $\boldsymbol{\xi}_{l}=\left[\left|\xi_{l x}\right\rangle,\left|\xi_{l y}\right\rangle,\left|\xi_{l z}\right\rangle\right]$ are defined as

$$
\begin{aligned}
\left|\xi_{l x}\right\rangle & =\left[\begin{array}{llllll}
0, & 1,-1, & 0, & 0, & 0, & 0
\end{array}\right]^{\top}, \\
\left|\xi_{l y}\right\rangle & =\left[\begin{array}{llllll}
0, & 0, & 0, & 1,-1, & 0, & 0
\end{array}\right]^{\top}, \\
\left|\xi_{l z}\right\rangle & =\left[\begin{array}{llllll}
0, & 0, & 0, & 0, & 0, & 1,-1
\end{array}\right]^{\top} .
\end{aligned}
$$

Vector-valued populations $\boldsymbol{h}_{l}$ are necessary due to the anti-symmetry of the electric tensor ${ }^{83}$. Their equilibrium state is defined as $^{84}$

$$
h_{l \alpha}^{e q}=w_{l}\left[b_{\alpha}+4 \xi_{l \beta}\left(u_{\beta} b_{\alpha}-b_{\beta} u_{\alpha}\right)\right],
$$

where $w_{0}=1 / 4, w_{1 \ldots 6}=1 / 8$ and $(\alpha, \beta) \in\{x, y, z\}^{2}$. Regarding magnetic boundary conditions, we follow the approach $\mathrm{in}^{82}$ that is built on the fact that the magnetic field can be computed as

$$
\boldsymbol{b}=\sum_{l=0}^{6} \boldsymbol{h}_{l} .
$$


Specifically, let us assume that one wants to enforce a certain magnetic field $\boldsymbol{b}^{\dagger}=\left[b_{x}^{\dagger}, b_{y}^{\dagger}, b_{z}^{\dagger}\right]$ to the bottom section of the considered domain. In this case, the only population to be assigned is $\boldsymbol{h}_{2}$, that is evaluated as

$$
\begin{aligned}
& h_{2 x}=b_{x}^{\dagger}-\sum_{l \neq 2} h_{l x}, \\
& h_{2 y}=b_{y}^{\dagger}-\sum_{l \neq 2} h_{l y}, \\
& h_{2 z}=b_{z}^{\dagger}-\sum_{l \neq 2} h_{l z} .
\end{aligned}
$$

\section{Appendix D: CMs-based color-gradient method}

Let us consider two immiscible fluids, namely, red and blue. The evolution of populations $f_{i}^{k}$ is

$$
f_{i}^{k}\left(\boldsymbol{x}+\boldsymbol{c}_{i}, t+1\right)=f_{i}^{k}(\boldsymbol{x}, t)+\Omega_{i}^{k}(\boldsymbol{x}, t),
$$

where $k=r$ for the red fluid, and $k=b$ for the blue one. Moreover, it is possible to define the total distribution functions as $f_{i}=f_{i}^{r}+f_{i}^{b}$. The collision process $\Omega_{i}^{k}$ is composed of three sub-stages:

$$
\Omega_{i}^{k}=\left(\Omega_{i}^{k}\right)^{(3)}\left[\left(\Omega_{i}^{k}\right)^{(1)}+\left(\Omega_{i}^{k}\right)^{(2)}\right],
$$

where $\left(\Omega_{i}^{k}\right)^{(1)},\left(\Omega_{i}^{k}\right)^{(2)}$ and $\left(\Omega_{i}^{k}\right)^{(3)}$ are the single-phase, perturbation and recoloring operators, respectively. Within the CM-based framework ${ }^{67}$, the single-phase collision operator can be written as

$$
\left(\Omega_{i}^{k}\right)^{(1)}=\left(\overline{\mathcal{T}}^{\top}\right)^{-1} \boldsymbol{\Lambda} \overline{\mathcal{T}}^{\top}\left(\left|f_{i}^{e q}\right\rangle-\left|f_{i}\right\rangle\right)+\left(\mathbf{I}-\frac{\boldsymbol{\Lambda}}{2}\right) \overline{\mathcal{T}}^{\top}\left|\mathcal{F}_{i}\right\rangle,
$$

with $\left|\mathcal{F}_{i}\right\rangle, \overline{\mathcal{T}}^{\top}$ and $\Lambda$ that obey Eqs. (9), (11) and (17), respectively. Macroscopic variables are given by

$$
\begin{aligned}
\rho_{k} & =\sum_{i} f_{i}^{k}, \\
\rho & =\sum_{k} \rho_{k}, \\
\rho \boldsymbol{u} & =\sum_{i} f_{i} \boldsymbol{c}_{i}+\frac{1}{2} \boldsymbol{F},
\end{aligned}
$$

where $\rho_{k}$ is the density of the fluid $k, \rho$ is the total mass density, $\boldsymbol{u}$ is the total momentum and $\boldsymbol{F}$ is a body force. Distributions relax to an enhanced local equilibrium ${ }^{95,99}$ that, by adopting sixth-order Hermite polynomials, read as follows:

$$
\begin{aligned}
f_{i}^{e q}(\rho, \boldsymbol{u})= & \rho\left\{\varphi_{i}+w_{i}\left[\frac{\boldsymbol{c}_{i} \cdot \boldsymbol{u}}{c_{s}^{2}}+\frac{1}{2 c_{s}^{4}}\left[\mathcal{H}_{i x x}^{(2)} u_{x}^{2}+\mathcal{H}_{i y y}^{(2)} u_{y}^{2}+\mathcal{H}_{i z z}^{(2)} u_{z}^{2}+2\left(\mathcal{H}_{i x y}^{(2)} u_{x} u_{y}+\mathcal{H}_{i x z}^{(2)} u_{x} u_{z}+\mathcal{H}_{i y z}^{(2)} u_{y} u_{z}\right)\right]\right.\right. \\
& +\frac{1}{2 c_{s}^{6}}\left[\mathcal{H}_{i x x y}^{(3)} u_{x}^{2} u_{y}+\mathcal{H}_{i x x z}^{(3)} u_{x}^{2} u_{z}+\mathcal{H}_{i x y y}^{(3)} u_{x} u_{y}^{2}+\mathcal{H}_{i x z z}^{(3)} u_{x} u_{z}^{2}+\mathcal{H}_{i y z z}^{(3)} u_{y} u_{z}^{2}+\mathcal{H}_{i y y z}^{(3)} u_{y}^{2} u_{z}+2 \mathcal{H}_{i x y z}^{(3)} u_{x} u_{y} u_{z}\right] \\
& +\frac{1}{4 c_{s}^{8}}\left[\mathcal{H}_{i x x y y}^{(4)} u_{x}^{2} u_{y}^{2}+\mathcal{H}_{i x x z z}^{(4)} u_{x}^{2} u_{z}^{2}+\mathcal{H}_{i y y z z}^{(4)} u_{y}^{2} u_{z}^{2}+2\left(\mathcal{H}_{i x y z z}^{(4)} u_{x} u_{y} u_{z}^{2}+\mathcal{H}_{i x y y z}^{(4)} u_{x} u_{y}^{2} u_{z}+\mathcal{H}_{i x x y z}^{(4)} u_{x}^{2} u_{y} u_{z}\right)\right] \\
& +\frac{1}{4 c_{s}^{10}}\left[\mathcal{H}_{i x x y z z}^{(5)} u_{x}^{2} u_{y} u_{z}^{2}+\mathcal{H}_{i x x y y z}^{(5)} u_{x}^{2} u_{y}^{2} u_{z}+\mathcal{H}_{i x y y z z}^{(5)} u_{x} u_{y}^{2} u_{z}^{2}\right] \\
& \left.\left.+\frac{1}{8 c_{s}^{12}} \mathcal{H}_{\text {ixxyyzz}}^{(6)} u_{x}^{2} u_{y}^{2} u_{z}^{2}\right]\right\}+\Phi_{i} .
\end{aligned}
$$

Here, quantities $\varphi_{i}$ and $\Phi_{i}$ are

$$
\varphi_{i}= \begin{cases}\bar{\alpha}, & \left|\boldsymbol{c}_{i}\right|^{2}=0, \\ 2(1-\bar{\alpha}) / 19, & \left|\boldsymbol{c}_{i}\right|^{2}=1, \\ (1-\bar{\alpha}) / 38, & \left|\boldsymbol{c}_{i}\right|^{2}=2, \\ (1-\bar{\alpha}) / 152, & \left|\boldsymbol{c}_{i}\right|^{2}=3,\end{cases}
$$

and

$$
\Phi_{i}= \begin{cases}-3 \bar{\nu}(\mathbf{u} \cdot \nabla \rho), & \left|\boldsymbol{c}_{i}\right|^{2}=0, \\ +16 \bar{\nu}\left(\mathbf{G}: \boldsymbol{c}_{i} \otimes \boldsymbol{c}_{i}\right), & \left|\boldsymbol{c}_{i}\right|^{2}=1, \\ +4 \bar{\nu}\left(\mathbf{G}: \boldsymbol{c}_{i} \otimes \boldsymbol{c}_{i}\right), & \left|\boldsymbol{c}_{i}\right|^{2}=2, \\ +1 \bar{\nu}\left(\mathbf{G}: \boldsymbol{c}_{i} \otimes \boldsymbol{c}_{i}\right), & \left|\boldsymbol{c}_{i}\right|^{2}=3,\end{cases}
$$

where $\otimes$ is the tensor (outer) product, and : stands for the contraction of index (Frobenius inner product). The tensor
$\mathbf{G}$ is defined as

$$
\mathbf{G}=\frac{1}{48}\left[\mathbf{u} \otimes \nabla \rho+(\mathbf{u} \otimes \nabla \rho)^{\top}\right],
$$

where gradients are computed by a second-order isotropic central scheme ${ }^{100} . \bar{\nu}$ is the kinematic viscosity interpolated by $^{94}$

$$
\frac{1}{\bar{\nu}}=\frac{1+\phi}{2} \frac{1}{\nu_{r}}+\frac{1-\phi}{2} \frac{1}{\nu_{b}} .
$$

To distinguish the two components, the order parameter $\phi$ is introduced, that is

$$
\phi=\left(\frac{\rho_{r}}{\rho_{r}^{0}}-\frac{\rho_{b}}{\rho_{b}^{0}}\right) /\left(\frac{\rho_{r}}{\rho_{r}^{0}}+\frac{\rho_{b}}{\rho_{b}^{0}}\right) .
$$

The values $\phi=1,-1$, and 0 correspond to a purely red fluid, a purely blue fluid, and the interface, respectively. To 
obtain a stable interface, the density ratio between the fluids must be taken into account as follows to obtain a stable interface $^{101}$ :

$$
\frac{\rho_{r}^{0}}{\rho_{b}^{0}}=\frac{1-\alpha_{b}}{1-\alpha_{r}}
$$

where the superscript " 0 " indicates the initial value of the density at the beginning of the simulation ${ }^{102}$. The pressure of the fluid is given as an isothermal equation of state:

$$
p=\rho\left(c_{s}^{k}\right)^{2}=\rho_{k} \frac{9(1-\bar{\alpha})}{19},
$$

for the D3Q27 lattice, where $c_{s}^{k}$ is the speed of sound of the fluid $k^{94}, \bar{\alpha}$ is interpolated by

$$
\bar{\alpha}=\frac{1+\phi}{2} \alpha_{r}+\frac{1-\phi}{2} \alpha_{b},
$$

with $\alpha_{b}=8 / 27$ and $c_{s}^{b}=1 / \sqrt{3}^{67}$.

Following $93,100,103$, the interfacial tension is modeled by the so-called perturbation operator:

$$
\left(\Omega_{i}\right)^{(2)}=\frac{A}{2}|\nabla \phi|\left[w_{i} \frac{\left(\boldsymbol{c}_{i} \cdot \nabla \phi\right)}{|\nabla \phi|^{2}}-B_{i}\right],
$$

where

$$
B_{i}=\left\{\begin{array}{cc}
-10 / 27, & \left|\boldsymbol{c}_{i}\right|^{2}=0 \\
+2 / 27, & \left|\boldsymbol{c}_{i}\right|^{2}=1 \\
+1 / 54, & \left|\boldsymbol{c}_{i}\right|^{2}=2 \\
+1 / 216, & \left|\boldsymbol{c}_{i}\right|^{2}=3
\end{array}\right.
$$

Eventually, the following recoloring operator is applied to promote phase segregation and maintain the interface:

$$
\begin{aligned}
& \left(\Omega_{i}^{r}\right)^{(3)}=\frac{\rho_{r}}{\rho} f_{i}+\gamma \frac{\rho_{r} \rho_{b}}{\rho^{2}} \cos \left(\theta_{i}\right) f_{i}^{e q}(\rho, \mathbf{0}), \\
& \left(\Omega_{i}^{b}\right)^{(3)}=\frac{\rho_{b}}{\rho} f_{i}-\gamma \frac{\rho_{r} \rho_{b}}{\rho^{2}} \cos \left(\theta_{i}\right) f_{i}^{e q}(\rho, \mathbf{0}),
\end{aligned}
$$

where $\gamma=0.7^{67,94,95}, \theta_{i}$ is the angle between $\nabla \phi$ and $\boldsymbol{c}_{i}$, which is defined by

$$
\cos \left(\theta_{i}\right)=\frac{\boldsymbol{c}_{i} \cdot \nabla \phi}{\left|\boldsymbol{c}_{i}\right||\nabla \phi|}
$$

\section{Appendix E: General MRT LBM}

In Section II, we derived a CMs-based LBM by defining a matrix $\overline{\mathcal{T}}$, allowing us to move from the velocity space to the CMs one (and vice versa). This matrix can be seen as the product of two contributions ${ }^{37,48,104}$

$$
\overline{\mathcal{T}}=\mathcal{S} \mathcal{T}
$$

The latter depends only on the chosen lattice, while the former accounts for velocity-dependent terms. In other words, the matrix $\mathcal{S}$ represents the effect of shifting the lattice direction by the local fluid velocity. Therefore, it plays the role of a shifting matrix, allowing us to move from the frame at rest to the co-moving one. Interestingly, the classical RMs-based MRT can be immediately derived by the present approach when the shifting matrix is disregarded (i.e., $\overline{\mathcal{T}}=\mathcal{T}$ ). Consistently, the methodology outlined in Section II can be interpreted as a general MRT-LBM, where the CMs-based approach degrades into the RMs-based one when $\mathcal{S}=\boldsymbol{I}$.

Let us define pre-collision, equilibrium and post-collision RMs as

$$
\begin{aligned}
\left|m_{i}\right\rangle & =\left[m_{0}, \ldots, m_{i}, \ldots, m_{26}\right]^{\top}, \\
\left|m_{i}^{e q}\right\rangle & =\left[m_{0}^{e q}, \ldots, m_{i}^{e q}, \ldots, m_{26}^{e q}\right]^{\top}, \\
\left|m_{i}^{\star}\right\rangle & =\left[m_{0}^{\star}, \ldots, m_{i}^{\star}, \ldots, m_{26}^{\star}\right]^{\top}
\end{aligned}
$$

respectively. Consistently with the CMs-based approach, RMs are computed by applying the transformation matrix $\mathcal{T}$ to the populations, that is

$$
\begin{aligned}
\left|m_{i}\right\rangle & =\mathcal{T}^{\top}\left|f_{i}\right\rangle, \\
\left|m_{i}^{e q}\right\rangle & =\mathcal{T}^{\top}\left|f_{i}^{e q}\right\rangle,
\end{aligned}
$$

By adopting the full set of Hermite polynomials, equilibrium RMs read as follows ${ }^{37}$

$$
\begin{aligned}
& m_{0}^{e q}=\rho, \\
& m_{1}^{e q}=\rho u_{x}, \\
& m_{2}^{e q}=\rho u_{y}, \\
& m_{3}^{e q}=\rho u_{z}, \\
& m_{4}^{e q}=\rho u_{x} u_{y}, \\
& m_{5}^{e q}=\rho u_{x} u_{z} \text {, } \\
& m_{6}^{e q}=\rho u_{y} u_{z} \text {, } \\
& m_{7}^{e q}=\rho\left(u_{x}^{2}-u_{y}^{2}\right), \\
& m_{8}^{e q}=\rho\left(u_{x}^{2}-u_{z}^{2}\right), \\
& m_{9}^{e q}=\rho\left(u_{x}^{2}+u_{y}^{2}+u_{z}^{2}+1\right), \\
& m_{10}^{e q}=\rho c_{s}^{2} u_{x}\left(3 u_{y}^{2}+3 u_{z}^{2}+2\right) \text {, } \\
& m_{11}^{e q}=\rho c_{s}^{2} u_{y}\left(3 u_{x}^{2}+3 u_{z}^{2}+2\right), \\
& m_{12}^{e q}=\rho c_{s}^{2} u_{z}\left(3 u_{x}^{2}+3 u_{y}^{2}+2\right) \text {, } \\
& m_{13}^{e q}=\rho u_{x}\left(u_{y}^{2}-u_{z}^{2}\right), \\
& m_{14}^{e q}=\rho u_{y}\left(u_{x}^{2}-u_{z}^{2}\right), \\
& m_{15}^{e q}=\rho u_{z}\left(u_{x}^{2}-u_{y}^{2}\right), \\
& m_{16}^{e q}=\rho u_{x} u_{y} u_{z} \\
& m_{17}^{e q}=\rho c_{s}^{2}\left[3\left(u_{x}^{2} u_{y}^{2}+u_{x}^{2} u_{z}^{2}+u_{y}^{2} u_{z}^{2}\right)+2\left(u_{x}^{2}+u_{y}^{2}+u_{z}^{2}\right)+1\right], \\
& m_{18}^{e q}=\rho c_{s}^{4}\left[9\left(u_{x}^{2} u_{y}^{2}+u_{x}^{2} u_{z}^{2}-u_{y}^{2} u_{z}^{2}\right)+6 u_{x}^{2}+1\right], \\
& m_{19}^{e q}=\rho c_{s}^{2}\left(u_{y}^{2}-u_{z}^{2}\right)\left(2 u_{x}^{2}+1\right) \text {, } \\
& m_{20}^{e q}=\rho c_{s}^{2} u_{y} u_{z}\left(3 u_{x}^{2}+1\right) \text {, } \\
& m_{21}^{e q}=\rho c_{s}^{2} u_{x} u_{z}\left(3 u_{y}^{2}+1\right) \text {, } \\
& m_{22}^{e q}=\rho c_{s}^{2} u_{x} u_{y}\left(3 u_{z}^{2}+1\right) \text {, } \\
& m_{23}^{e q}=\rho c_{s}^{4} u_{x}\left(3 u_{y}^{2}+1\right)\left(3 u_{z}^{2}+1\right), \\
& m_{24}^{e q}=\rho c_{s}^{4} u_{y}\left(3 u_{x}^{2}+1\right)\left(3 u_{z}^{2}+1\right), \\
& m_{25}^{e q}=\rho c_{s}^{4} u_{z}\left(3 u_{x}^{2}+1\right)\left(3 u_{y}^{2}+1\right) \text {, } \\
& m_{26}^{e q}=\rho c_{s}^{6}\left(3 u_{x}^{2}+1\right)\left(3 u_{y}^{2}+1\right)\left(3 u_{z}^{2}+1\right) \text {. }
\end{aligned}
$$

One can immediately observe that all the equilibrium RMs are non-zero. Moreover, all show a dependence on the fluid velocity. This is explained by the fact that RMs are linear combinations of (non-zero) equilibrium CMs multiplied by powers of the velocity components ${ }^{37}$. In other words, it is not 
possible to obtain Galilean invariant equilibrium moments when RMs are considered, even if Hermite polynomial up to the sixth-order are adopted. This has an impact on the numerical stability of the resulting RM-LBM even if its macroscopic behavior remains unchanged ${ }^{37}$.

The post-collision RMs can be written as

$$
\left|m_{i}^{\star}\right\rangle=(\mathbf{I}-\boldsymbol{\Lambda}) \mathcal{T}^{\top}\left|f_{i}\right\rangle+\boldsymbol{\Lambda} \mathcal{T}^{\top}\left|f_{i}^{e q}\right\rangle,
$$

or

$$
\left|m_{i}^{\star}\right\rangle=(\mathbf{I}-\boldsymbol{\Lambda})\left|m_{i}\right\rangle+\boldsymbol{\Lambda}\left|m_{i}^{e q}\right\rangle,
$$

and read as follows

$$
\begin{aligned}
& m_{0}^{\star}=\rho, \\
& m_{1}^{\star}=\rho u_{x}, \\
& m_{2}^{\star}=\rho u_{y}, \\
& m_{3}^{\star}=\rho u_{z} \text {, } \\
& m_{4}^{\star}=(1-\omega) m_{4}+\omega \rho u_{x} u_{y}, \\
& m_{5}^{\star}=(1-\omega) m_{5}+\omega \rho u_{x} u_{z}, \\
& m_{6}^{\star}=(1-\omega) m_{6}+\omega \rho u_{y} u_{z} \text {, } \\
& m_{7}^{\star}=(1-\omega) m_{7}+\omega \rho\left(u_{x}^{2}-u_{y}^{2}\right) \text {, } \\
& m_{8}^{\star}=(1-\omega) m_{8}+\omega \rho\left(u_{x}^{2}-u_{z}^{2}\right) \text {, } \\
& m_{9}^{\star}=\rho\left(u_{x}^{2}+u_{y}^{2}+u_{z}^{2}+1\right), \\
& m_{10}^{\star}=\rho c_{s}^{2} u_{x}\left(3 u_{y}^{2}+3 u_{z}^{2}+2\right) \text {, } \\
& m_{11}^{\star}=\rho c_{s}^{2} u_{y}\left(3 u_{x}^{2}+3 u_{z}^{2}+2\right) \text {, } \\
& m_{12}^{\star}=\rho c_{s}^{2} u_{z}\left(3 u_{x}^{2}+3 u_{y}^{2}+2\right) \text {, } \\
& m_{13}^{\star}=\rho u_{x}\left(u_{y}^{2}-u_{z}^{2}\right) \text {, } \\
& m_{14}^{\star}=\rho u_{y}\left(u_{x}^{2}-u_{z}^{2}\right) \text {, } \\
& m_{15}^{\star}=\rho u_{z}\left(u_{x}^{2}-u_{y}^{2}\right) \text {, } \\
& m_{16}^{\star}=\rho u_{x} u_{y} u_{z} \text {, } \\
& m_{17}^{\star}=\rho c_{s}^{2}\left[3\left(u_{x}^{2} u_{y}^{2}+u_{x}^{2} u_{z}^{2}+u_{y}^{2} u_{z}^{2}\right)+2\left(u_{x}^{2}+u_{y}^{2}+u_{z}^{2}\right)+1\right] \text {, } \\
& m_{18}^{\star}=\rho c_{s}^{4}\left[9\left(u_{x}^{2} u_{y}^{2}+u_{x}^{2} u_{z}^{2}-u_{y}^{2} u_{z}^{2}\right)+6 u_{x}^{2}+1\right] \text {, } \\
& m_{19}^{\star}=\rho c_{s}^{2}\left(u_{y}^{2}-u_{z}^{2}\right)\left(2 u_{x}^{2}+1\right) \text {, } \\
& m_{20}^{\star}=\rho c_{s}^{2} u_{y} u_{z}\left(3 u_{x}^{2}+1\right) \text {, } \\
& m_{21}^{\star}=\rho c_{s}^{2} u_{x} u_{z}\left(3 u_{y}^{2}+1\right) \text {, } \\
& m_{22}^{\star}=\rho c_{s}^{2} u_{x} u_{y}\left(3 u_{z}^{2}+1\right) \text {, } \\
& m_{23}^{\star}=\rho c_{s}^{4} u_{x}\left(3 u_{y}^{2}+1\right)\left(3 u_{z}^{2}+1\right) \text {, } \\
& m_{24}^{\star}=\rho c_{s}^{4} u_{y}\left(3 u_{x}^{2}+1\right)\left(3 u_{z}^{2}+1\right) \text {, } \\
& m_{25}^{\star}=\rho c_{s}^{4} u_{z}\left(3 u_{x}^{2}+1\right)\left(3 u_{y}^{2}+1\right) \text {, } \\
& m_{26}^{\star}=\rho c_{s}^{6}\left(3 u_{x}^{2}+1\right)\left(3 u_{y}^{2}+1\right)\left(3 u_{z}^{2}+1\right) \text {, }
\end{aligned}
$$

where

$$
\begin{aligned}
& m_{4}=\sum_{i} f_{i} c_{i x} c_{i y}, \\
& m_{5}=\sum_{i} f_{i} c_{i x} c_{i z}, \\
& m_{6}=\sum_{i} f_{i} c_{i y} c_{i z}, \\
& m_{7}=\sum_{i} f_{i}\left(c_{i x}^{2}-c_{i y}^{2}\right), \\
& m_{8}=\sum_{i} f_{i}\left(c_{i x}^{2}-c_{i z}^{2}\right) .
\end{aligned}
$$

Again, all post-collision RMs are non-zero and also show a dependence on the velocity (except for the first conserved moment, $\left.m_{0}\right)$. Eventually, the post-collision populations are reconstructed as

$$
\left|f_{i}^{\star}\right\rangle=\left(\mathcal{T}^{\top}\right)^{-1}\left|m_{i}^{\star}\right\rangle
$$

and streamed (see Eq. 4).

${ }^{1}$ S. Succi, The Lattice Boltzmann Equation for Fluid Dynamics and Beyond (Clarendon, 2001).

${ }^{2}$ S. Succi, "Lattice Boltzmann 2038," Europhys. Lett. 109, 50001-50007 (2015).

${ }^{3}$ S. Succi, "Chimaera simulation of complex states of flowing matter," Phil. Trans. R. Soc. A 374 (2016).

${ }^{4}$ T. Krüger, H. Kusumaatmaja, A. Kuzmin, O. Shardt, G. Silva, and E. M. Viggen, The Lattice Boltzmann Method: Principles and Practice (Springer, 2016).

${ }^{5}$ S. Succi, The Lattice Boltzmann Equation: For Complex States of Flowing Matter (Oxford University Press, 2018).

${ }^{6}$ Z. Chen, C. Shu, and D. Tan, "Highly accurate simplified lattice boltzmann method," Physics Fluids 30, 103605 (2018), https://doi.org/10.1063/1.5050185.

${ }^{7}$ D. Wang, D. Tan, and N. Phan-Thien, "A lattice boltzmann method for simulating viscoelastic drops," Physics Fluids 31, 073101 (2019), https://doi.org/10.1063/1.5100327.

${ }^{8}$ X. Liu, Z. Chai, and B. Shi, "A phase-field-based lattice boltzmann modeling of two-phase electro-hydrodynamic flows," Physics Fluids 31, 092103 (2019), https://doi.org/10.1063/1.5119891.

${ }^{9}$ D. Maggiolo, F. Picano, and M. Guarnieri, "Flow and dispersion in anisotropic porous media: A lattice-boltzmann study," Physics Fluids 28, 102001 (2016), https://doi.org/10.1063/1.4963766.

${ }^{10}$ L. Zhang, A. S. Jebakumar, and J. Abraham, "Lattice boltzmann method simulations of stokes number effects on particle motion in a channel flow," Physics Fluids 28, 063306 (2016), https://doi.org/10.1063/1.4953800.

${ }^{11} \mathrm{P}$. Bhatnagar, E. Gross, and M. Krook, "A model for collision processes in gases. I. small amplitude processes in charged and neutral one-component systems," Phys. Rev. 94, 511 (1954).

${ }^{12} \mathrm{X}$. Shan, X.-F. Yuan, and H. Chen, "Kinetic theory representation of hydrodynamics: a way beyond the navier-stokes equation," J. Fluid Mech. 550, 413 (2006).

${ }^{13}$ I. V. Karlin, A. N. Gorban, S. Succi, and V. Boffi, "Maximum entropy principle for lattice kinetic equations," Phys. Rev. Lett. 81, 6-9 (1998)

${ }^{14}$ B. M. Boghosian, J. Yepez, P. V. Coveney, and A. Wager, "Entropic lattice Boltzmann methods," Proc. Royal Soc. A 457, 717-766 (2001).

${ }^{15}$ S. S. Chikatamarla, C. E. Frouzakis, I. V. Karlin, A. G. Tomboulides, and K. B. Boulouchos, "Lattice Boltzmann method for direct numerical simulation of turbulent flows," J. Fluid Mech. 656, 298-308 (2010).

${ }^{16}$ K. Huang, Statistical Mechanics, 2nd Edition, 2nd ed. (Wiley, 1987). ${ }^{17}$ O. Malaspinas, M. Deville, and B. Chopard, "Towards a physical interpretation of the entropic lattice Boltzmann method," Phys. Rev. E 78, 066705 (2008).

${ }^{18}$ I. V. Karlin, F. Bösch, and S. S. Chikatamarla, "Gibbs' principle for the lattice-kinetic theory of fluid dynamics," Phys. Rev. E 90, 031302 (2014).

${ }^{19}$ M. Atif, P. K. Kolluru, C. Thantanapally, and S. Ansumali, "Essentially entropic lattice boltzmann model," Phys. Rev. Lett. 119, 240602 (2017).

${ }^{20}$ M. A. Mazloomi, S. S. Chikatamarla, and I. V. Karlin, "Entropic lattice Boltzmann method for multiphase flows: Fluid-solid interfaces," Phys. Rev. E 92, 023308 (2015).

${ }^{21}$ N. Frapolli, Entropic lattice Boltzmann models for thermal and compressible flows, Ph.D. thesis, ETH-Zürich (2017).

${ }^{22}$ F. Bösch, Entropic Lattice Boltzmann Models for Fluid Dynamics, Ph.D. thesis, ETH-Zürich (2017).

${ }^{23}$ B. Dorschner, Entropic Lattice Boltzmann Method for Complex Flows, Ph.D. thesis, ETH-Zürich (2018).

${ }^{24}$ C. Flint and G. Vahala, "Partial entropic stabilization of lattice boltzmann magnetohydrodynamics," Phys. Rev. E 97, 013302 (2018). 
${ }^{25} \mathrm{~F}$. Higuera and J. Jimenez, "Boltzmann approach to lattice gas simulations," Europhys. Lett. 9, 663 (1989).

${ }^{26}$ R. Benzi, S. Succi, and M. Vergassola, "The lattice Boltzmann equation: Theory and applications," Phys. Rep. 222, 145-197 (1992).

${ }^{27}$ D. d'Humières, "Generalized lattice-Boltzmann equations," Prog. Astronaut. Aeronaut. 159, 450-458 (1992).

${ }^{28}$ P. Lallemand and L.-S. Luo, "Theory of the lattice Boltzmann method: Dispersion, dissipation, isotropy, galilean invariance, and stability," Phys. Rev. E 61, 6546-6562 (2000).

${ }^{29}$ P. J. Dellar, "Nonhydrodynamic modes and a priori construction of shallow water lattice Boltzmann equations," Phys. Rev. E 65, 036309 (2002).

${ }^{30} \mathrm{~J}$. Latt and B. Chopard, "Lattice boltzmann method with regularized pre-collision distribution functions," Math. Comput. Simulat. 72, $165-168$ (2006).

${ }^{31}$ S. Marié, D. Ricot, and P. Sagaut, "Comparison between lattice boltzmann method and navier-stokes high order schemes for computational aeroacoustics," J. Comput. Phys. 228, 1056-1070 (2009).

${ }^{32}$ H. Xu and P. Sagaut, "Optimal low-dispersion low-dissipation LBM schemes for computational aeroacoustics," J. Comput. Phys. 230, $5353-5382$ (2011).

${ }^{33}$ Y. H. Qian and S. A. Orszag, "Lattice BGK models for the Navier-Stokes equation: Nonlinear deviation in compressible regimes," Europhys. Lett. 21, 255 (1993).

${ }^{34}$ X. B. Nie, X. Shan, and H. Chen, "Galilean invariance of lattice boltzmann models," Europhys. Lett. 81, 34005 (2008).

${ }^{35} \mathrm{C}$. Coreixas, High-order extension of the recursive regularized lattice Boltzmann method, Ph.D. thesis, INP Toulouse (2018).

${ }^{36} \mathrm{M}$. Geier, A. Greiner, and J. Korvink, "Cascaded digital lattice Boltzmann automata for high Reynolds number flow," Phys. Rev. E 73, 066705 (2006).

${ }^{37}$ C. Coreixas, B. Chopard, and J. Latt, "Comprehensive comparison of collision models in the lattice boltzmann framework: Theoretical investigations," Phys. Rev. E 100, 033305 (2019).

${ }^{38} \mathrm{M}$. Geier, A. Greiner, and J. Korvink, "Properties of the cascaded lattice Boltzmann automaton," Int. J. Mod. Phys. C 18, 455-462 (2007).

${ }^{39} \mathrm{M}$. Geier, "De-aliasing and stabilization formalism of the cascaded lattice Boltzmann automaton for under-resolved high Reynolds number flow," Int. J. Numer. Meth. Fl. 56, 1249-1254 (2008).

${ }^{40} \mathrm{P}$. Asinari, "Generalized local equilibrium in the cascaded lattice Boltzmann method," Phys. Rev. E 78, 016701 (2008).

${ }^{41} \mathrm{M}$. Geier, A. Greiner, and J. Korvink, "A factorized central moment lattice Boltzmann method," Eur. Phys. J-Spec. Top. 171, 55-61 (2009).

${ }^{42}$ M. Geier, M. Schönherr, A. Pasquali, and M. Krafczyk, "The cumulant lattice Boltzmann equation in three dimensions: Theory and validation," Comput. Math. Appl. 70, 507-547 (2015).

${ }^{43} \mathrm{~A}$. De Rosis and E. Lévêque, "Central-moment lattice boltzmann schemes with fixed and moving immersed boundaries," Comput. Math. Appl. 72, 1616-1628 (2016).

${ }^{44}$ M. Geier, A. Pasquali, and M. Schönherr, "Parametrization of the cumulant lattice boltzmann method for fourth order accurate diffusion part i: Derivation and validation," J. Comput. Phys. 348, 862-888 (2017).

${ }^{45}$ M. Geier, A. Pasquali, and M. Schönherr, "Parametrization of the cumulant lattice boltzmann method for fourth order accurate diffusion part ii: Application to flow around a sphere at drag crisis," J. Comput. Phys. 348, 889-898 (2017).

${ }^{46} \mathrm{M}$. Geier and A. Pasquali, "Fourth order galilean invariance for the lattice boltzmann method," Comput. Fluids 166, 139-151 (2018).

${ }^{47} \mathrm{~L}$. Fei and K. H. Luo, "Consistent forcing scheme in the cascaded lattice boltzmann method," Phys. Rev. E 96, 053307 (2017).

${ }^{48} \mathrm{~L}$. Fei, K. H. Luo, and Q. Li, "Three-dimensional cascaded lattice boltzmann method: Improved implementation and consistent forcing scheme," Phys. Rev. E 97, 053309 (2018).

${ }^{49}$ N. Shah, P. Dhar, S. K. Chinige, M. Geier, and A. Pattamatta, "Cascaded collision lattice boltzmann model (clbm) for simulating fluid and heat transport in porous media," Numer. Heat Tr. B-Fund. 72, 211-232 (2017).

${ }^{50}$ C. S. Kumar, S. Mohankumar, M. Geier, and A. Pattamatta, "Numerical investigations on convective heat transfer enhancement in jet impingement due to the presence of porous media using cascaded lattice boltzmann method," Int. J. Therm. Sci. 122,
201-217 (2017).

${ }^{51}$ K. V. Sharma, R. Straka, and F. W. Tavares, "New cascaded thermal lattice boltzmann method for simulations of advection-diffusion and convective heat transfer," Int. J. Therm. Sci. 118, 259-277 (2017).

${ }^{52}$ L. Fei and K. H. Luo, "Cascaded lattice boltzmann method for thermal flows on standard lattices," Int. J. Therm. Sci. 132, 368-377 (2018).

${ }^{53}$ L. Fei and K. H. Luo, "Cascaded lattice boltzmann method for incompressible thermal flows with heat sources and general thermal boundary conditions," Comput. Fluids 165, 89-95 (2018).

${ }^{54}$ H. Safari, M. Krafczyk, and M. Geier, "A lattice boltzmann model for thermal compressible flows at low mach numbers beyond the boussinesq approximation," Comput. Fluids (2018).

${ }^{55} \mathrm{~K}$. Premnath and S. Banerjee, "Incorporating forcing terms in cascaded lattice Boltzmann approach by method of central moments," Phys. Rev. E 80, 036702 (2009).

${ }^{56} \mathrm{~K}$. Premnath and S. Banerjee, "On the three-dimensional central moment lattice Boltzmann method," J. Stat. Phys. 143, 747-794 (2011).

${ }^{57}$ Y. Ning, K. N. Premnath, and D. V. Patil, "Numerical study of the properties of the central moment lattice boltzmann method," Int. J. Numer. Meth. Fl. 82, 59-90 (2016).

${ }^{58}$ F. Hajabdollahi and K. N. Premnath, "Improving the low mach number steady state convergence of the cascaded lattice boltzmann method by preconditioning," Comput. Math. Appl. (2017).

${ }^{59} \mathrm{M}$. Chávez-Modena, E. Ferrer, and G. Rubio, "Improving the stability of multiple-relaxation lattice boltzmann methods with central moments," Comput. Fluids 172, 397 - 409 (2018).

${ }^{60}$ A. De Rosis, "Non-orthogonal central moments relaxing to a discrete equilibrium: A d2q9 lattice boltzmann model," Europhys. Lett. 116, 44003 (2016).

${ }^{61}$ A. De Rosis, "Nonorthogonal central-moments-based lattice boltzmann scheme in three dimensions," Phys. Rev. E 95, 013310 (2017).

${ }^{62}$ A. De Rosis, "Alternative formulation to incorporate forcing terms in a lattice boltzmann scheme with central moments," Phys. Rev. E 95, 023311 (2017).

${ }^{63} \mathrm{~A}$. De Rosis, "Central-moments-based lattice boltzmann schemes with force-enriched equilibria," Europhys. Lett. 117, 34003 (2017).

${ }^{64}$ A. De Rosis, "Preconditioned lattice boltzmann method for steady flows: A noncascaded central-moments-based approach," Phys. Rev. E 96, 063308 (2017).

${ }^{65}$ A. De Rosis, "A central moments-based lattice boltzmann scheme for shallow water equations," Comput. Method. Appl. M. 319, 379-392 (2017).

${ }^{66}$ A. De Rosis, E. Lévêque, and R. Chahine, "Advanced lattice boltzmann scheme for high-reynolds-number magneto-hydrodynamic flows," J. Turbul. 19, 446-462 (2018).

${ }^{67} \mathrm{~S}$. Saito, A. De Rosis, A. Festuccia, A. Kaneko, Y. Abe, and K. Koyama, "Color-gradient lattice boltzmann model with nonorthogonal central moments: Hydrodynamic melt-jet breakup simulations," Phys. Rev. E 98, 013305 (2018).

${ }^{68} \mathrm{O}$. Malaspinas, "Increasing stability and accuracy of the lattice boltzmann scheme: recursivity and regularization," arXiv preprint arXiv:1505.06900 (2015).

${ }^{69}$ C. Coreixas, G. Wissocq, G. Puigt, J.-F. Boussuge, and P. Sagaut, "Recursive regularization step for high-order lattice boltzmann methods," Phys. Rev. E 96, 033306 (2017).

${ }^{70} \mathrm{~A}$. De Rosis and K. H. Luo, "Role of higher-order hermite polynomials in the central-moments-based lattice boltzmann framework," Phys. Rev. E 99, 013301 (2019).

${ }^{71} \mathrm{H}$. Grad, "On the kinetic theory of rarefied gases," Commun. Pure Appl. Maths 2, 331-407 (1949).

${ }^{72} \mathrm{X}$. Shan and X. He, "Discretization of the velocity space in the solution of the Boltzmann equation," Phys. Rev. Lett. 80, 65-68 (1998).

${ }^{73} \mathrm{Z}$. Guo, C. Zheng, and B. Shi, "Discrete lattice effects on the forcing term in the lattice boltzmann method," Phys. Rev. E 65, 046308 (2002).

${ }^{74} \mathrm{Z}$. Guo and C. Zheng, "Analysis of lattice boltzmann equation for microscale gas flows: relaxation times, boundary conditions and the knudsen layer," Int. J. Comput. Fluid D. 22, 465-473 (2008).

${ }^{75} \mathrm{X}$. He, S. Chen, and G. D. Doolen, "A novel thermal model for the lattice boltzmann method in incompressible limit," Journal of Computational Physics 146, 282-300 (1998). 
${ }^{76}$ R. Huang, H. Wu, and N. A. Adams, "Eliminating cubic terms in the pseudopotential lattice Boltzmann model for multiphase flow," Phys. Rev. E 97, 053308 (2018).

${ }^{77}$ D. d'Humières, "Multiple-relaxation-time lattice Boltzmann models in three dimensions," Philos. T. R. Soc. A 360, 437-451 (2002).

${ }^{78}$ S. A. Hosseini, C. Coreixas, N. Darabiha, and D. Thévenin, "Stability of the lattice kinetic scheme and choice of the free relaxation parameter," Phys. Rev. E 99, 063305 (2019).

${ }^{79}$ See Supplemental Material at [D3Q27_ CentralMomentsWithForcingScheme.m] for performing all the symbolic manipulations to easily compute all formulas required for the implementation (the transformation matrix, the post-collision CMs, etc).

${ }^{80}$ J. Latt, B. Chopard, O. Malaspinas, M. Deville, and A. Michler, "Straight velocity boundaries in the lattice Boltzmann method," Phys. Rev. E 77, 056703 (2008).

${ }^{81}$ O. Malaspinas, N. Fietier, and M. Deville, "Lattice boltzmann method for the simulation of viscoelastic fluid flows," J. Non-Newton. Fluid 165, 1637-1653 (2010).

${ }^{82} \mathrm{P}$. J. Dellar, "Moment-based boundary conditions for lattice boltzmann magnetohydrodynamics," in Numerical Mathematics and Advanced Applications 2011 (Springer, 2013) pp. 83-90.

${ }^{83}$ P. J. Dellar, "Lattice kinetic schemes for magnetohydrodynamics," J. Comput. Phys. 179, 95-126 (2002).

${ }^{84}$ M. Pattison, K. Premnath, N. Morley, and M. Abdou, "Progress in lattice boltzmann methods for magnetohydrodynamic flows relevant to fusion applications," Fusion Eng. Des. 83, 557-572 (2008).

${ }^{85} \mathrm{~S}$. A. Orszag and C.-M. Tang, "Small-scale structure of two-dimensional magnetohydrodynamic turbulence," J. Fluid Mech. 90, 129-143 (1979).

${ }^{86}$ P. Mininni, A. Pouquet, and D. Montgomery, "Small-scale structures in three-dimensional magnetohydrodynamic turbulence," Phys. Rev. Lett. 97, 244503 (2006).

${ }^{87}$ J. M. Greene, "Geometrical properties of three-dimensional reconnecting magnetic fields with nulls," J. Geophys. Res.-Space $\mathbf{9 3}$, 8583-8590 (1988).

${ }^{88} \mathrm{X}$. Shan and H. Chen, "Lattice boltzmann model for simulating flows with multiple phases and components," Phys. Rev. E 47, 1815 (1993).

${ }^{89}$ O. Schenk and K. Gärtner, "Solving unsymmetric sparse systems of linear equations with PARDISO," Future Generation Computer Systems 20, 475-487 (2004).

${ }^{90}$ S. Kolodziej, M. A. M. Bullock, J. David, T. A. Davis, M. Henderson, $\mathrm{Y}$. Hu, and R. Sandstrom, "The SuiteSparse matrix collection website interface," Journal of Open Source Software 35 (2019).

${ }^{91}$ C. Ashcraft and R. G. Grimes, "SPOOLES: An object-oriented sparse matrix library." in PPSC (1999).

${ }^{92}$ M. Latva-Kokko and D. H. Rothman, "Diffusion properties of gradient-based lattice boltzmann models of immiscible fluids," Phys. Rev. E 71, 056702 (2005).

${ }^{93}$ T. Reis and T. N. Phillips, "Lattice boltzmann model for simulating immiscible two-phase flows," Journal of Physics A: Mathematical and Theoretical 40, 4033-4053 (2007).

${ }^{94}$ S. Leclaire, M. Reggio, and J.-Y. Trepanier, "Numerical evaluation of two recoloring operators for an immiscible two-phase flow lattice boltzmann model," Applied Mathematical Modelling 36, 2237 2252 (2012).

${ }^{95}$ S. Saito, Y. Abe, and K. Koyama, "Lattice boltzmann modeling and simulation of liquid jet breakup," Phys. Rev. E 96, 013317 (2017).

${ }^{96} \mathrm{X}$. He, R. Zhang, S. Chen, and G. D. Doolen, "On the three-dimensional rayleigh-taylor instability," Phys. Fluids 11 1143-1152 (1999)

${ }^{97}$ N. Wang, H. Liu, and C. Zhang, "Three-dimensional phase-field lattice boltzmann model for incompressible multiphase flows," J. Comput. Sci. 17, 340 - 356 (2016), discrete Simulation of Fluid Dynamics 2015.

${ }^{98} \mathrm{H}$. G. Lee and J. Kim, "Numerical simulation of the three-dimensional rayleigh-taylor instability," Comput. Math. Appl. 66, 1466 - 1474 (2013).

${ }^{99}$ S. Leclaire, N. Pellerin, M. Reggio, and J.-Y. Trepanier, "Enhanced equilibrium distribution functions for simulating immiscible multiphase flows with variable density ratios in a class of lattice boltzmann models," Int. J. Multiphas. Flow 57, 159-168 (2013)

${ }^{100}$ H. Liu, A. J. Valocchi, and Q. Kang, "Three-dimensional lattice boltzmann model for immiscible two-phase flow simulations," Phys. Rev. E 85, 046309 (2012).

${ }^{101}$ D. Grunau, S. Chen, and K. Eggert, "A lattice boltzmann model for multiphase fluid flows," Phys Fluids A-Fluid 5, 2557-2562 (1993).

${ }^{102}$ S. Leclaire, A. Parmigiani, O. Malaspinas, B. Chopard, and J. Latt, "Generalized three-dimensional lattice boltzmann color-gradient method for immiscible two-phase pore-scale imbibition and drainage in porous media," Phys. Rev. E 95, 033306 (2017).

103 J. Brackbill, D. Kothe, and C. Zemach, "A continuum method for modeling surface tension," J. Comput. Phys. 100, 335 - 354 (1992).

${ }^{104}$ F. Dubois, T. Février, and B. Graille, "Lattice Boltzmann schemes with relative velocities," Commun. Comput. Phys. 17, 1088-1112 (2015). 